\title{
Design of a very high-resolution small animal PET scanner using a silicon scatter detector insert
}

\author{
Sang-June Park ${ }^{1}$, W Leslie Rogers ${ }^{2,3}$ and Neal H Clinthorne ${ }^{3}$ \\ ${ }^{1}$ Department of Nuclear Engineering and Radiological Sciences, University of Michigan, \\ Ann Arbor, MI 48109, USA \\ 2 Department of Biomedical Engineering, University of Michigan, Ann Arbor, MI 48109, USA \\ ${ }^{3}$ Division of Nuclear Medicine, University of Michigan, Ann Arbor, MI 48109, USA \\ E-mail: sangjune@umich.edu
}

Received 22 September 2006, in final form 12 March 2007

Published 17 July 2007

Online at stacks.iop.org/PMB/52/4653

\begin{abstract}
A small animal positron emission tomography (PET) instrument using a high-resolution solid-state detector insert in a conventional PET system was investigated for its potential to achieve sub-millimeter spatial resolution for mouse imaging. Monte Carlo simulations were used to estimate the effect of detector configurations (thickness, length and radius) on sensitivity. From this initial study, a PET system having an inner cylindrical silicon detector $(4 \mathrm{~cm}$ ID, $4 \mathrm{~cm}$ length and $1.6 \mathrm{~cm}$ thickness composed of 16 layers of $300 \mu \mathrm{m} \times$ $300 \mu \mathrm{m} \times 1 \mathrm{~mm}$ pads), for scattering, surrounded by an outer cylindrical BGO scintillation detector $(17.6 \mathrm{~cm} \mathrm{ID,} 16 \mathrm{~cm}$ length and $2 \mathrm{~cm}$ thickness segmented into $3 \mathrm{~mm} \times 3 \mathrm{~mm} \times 20 \mathrm{~mm}$ crystals), for capture was evaluated in detail. In order to evaluate spatial resolution, sensitivity and image quality of the PET system, 2D images of multiple point and cylinder sources were reconstructed with the simulation data including blurring from positron range and annihilation photon acollinearity using filtered backprojection (FBP). Simulation results for ${ }^{18} \mathrm{~F}$ demonstrate $340 \mu \mathrm{m}$ FWHM at the center of the field of view with $1.0 \%$ sensitivity from the coincidence of single scattering events in both silicon detectors and $1.0 \mathrm{~mm}$ FWHM with $9.0 \%$ sensitivity from the coincidence of single scattering in the silicon and full energy absorption of the second photon in the BGO detector.
\end{abstract}

(Some figures in this article are in colour only in the electronic version)

\section{Introduction}

For metabolic and genetic studies, research on disease mechanisms, and radiotracer development for human applications, mice are often used as experimental animals. And 
while PET is desirable for its ability to map function, the 1-2 mm FWHM spatial resolution typical of current generation animal imagers renders investigation on small-sized organs such as the thyroid $(\sim 1-7 \mu \mathrm{l}$ total volume) and adrenal $(3-40 \mu \mathrm{l})$ as well as small functional regions in brain (400-800 $\mu \mathrm{l})$ and kidney (100-500 $\mu \mathrm{l})$ challenging. Existing devices based on scintillation detectors have difficulty achieving the resolution limit imposed by positron range and annihilation photon acollinearity both because of inherent resolution and sensitivity losses resulting from intra-detector Compton and coherent scatter, and because of poor (or no) depth-of-interaction (DOI) resolution coupled with the penetrating nature of annihilation photons.

On the scale of PET instruments for human subjects where detector elements have widths of 4-5 mm, inter-crystal Compton scatter does not appear to have a large effect on performance. The situation changes, however, as the width of the crystal elements is reduced below $\sim 2 \mathrm{~mm}$. While spatial resolution - as measured by the FWHM or even FWTM of the coincidence response-continues to improve, an increasing fraction of events are assigned to the wrong line of response (LOR). Long tails on the distribution of incorrectly assigned events introduce significant noise and contrast loss in the image essentially resulting in an intrinsic resolutionsensitivity tradeoff.

Individual-channel readout systems including Sherbrooke small animal PET (Michaud et al 2004) have been designed to avoid the scatter problem, even though such a system requires the production cost and complexity due to more electronic channels for readout. While systems having individual detector readout can circumvent the scatter problem to some degree, the ultimate success depends upon the energy resolution and electronic noise of the detectors as well as the Doppler broadening of the scintillation materials. Instruments based on scintillation detectors and avalanche photodiode (APD) detectors (with the possible exception of the new lanthanum halide scintillators) are likely to have more difficulty in sorting out multiple interactions than the solid-state detector devices such as silicon, cadmium zinc telluride (CZT) and gallium arsenide (GaAs) due to the limitations associated with the larger energy uncertainties.

The DOI uncertainty resulting from the penetrating nature of $511 \mathrm{keV}$ annihilation photons in the detector ring introduces additional blurring. The further the source is located from the center of the field-of-view (FOV) the more significant the blurring. The effect, known as radial elongation, parallax error, or radial astigmatism, can be removed by measuring the DOI. By using phoswich detector technology, the NIH ATLAS (Seidel et al 2003) and GE eXplore Vista have been constructed to measure DOI. DOI can also be compensated in the image reconstruction algorithm such as maximum a posteriori (MAP) that accurately model the PET system response (Qi et al 1998). Numerous additional methods have been proposed to determine DOI (Miyaoka et al 1998, Huber et al 2001, Shao et al 2002, Burr et al 2004, Dokhale et al 2006). Nevertheless, DOI resolution measured using these methods is typically 3-6 mm. Moreover, the degree of DOI deblurring in the reconstruction must inevitably be traded off against noise amplification. The end result is that DOI uncertainty still contributes significant blurring to high-resolution images.

A PET scanner capable of sub-millimeter spatial resolution must have solutions for both inter-crystal scatter and the DOI uncertainty problems. Moreover, such an instrument cannot forgo the need to maintain acceptable energy and time resolutions as well as having low deadtime and high sensitivity. No existing PET instrument meets all these requirements.

We have examined a potential solution to these problems using a somewhat different approach to detecting annihilation radiation that is capable of achieving very high-spatialresolution images. Reconstructed image resolution, sensitivity, count rate performance were evaluated in this preliminary assessment of the proposed detector system. 


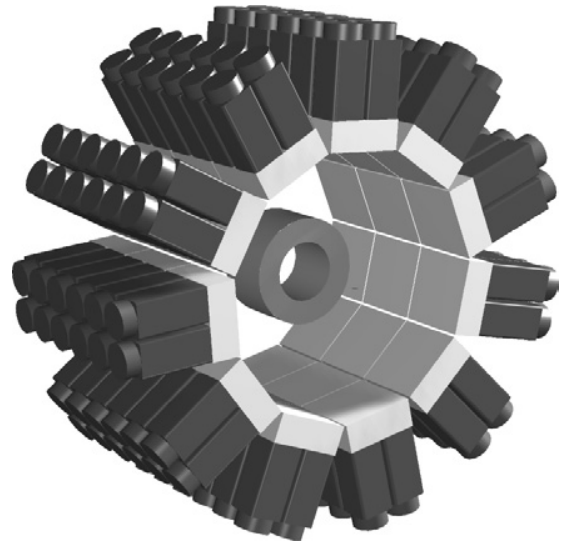

(a)

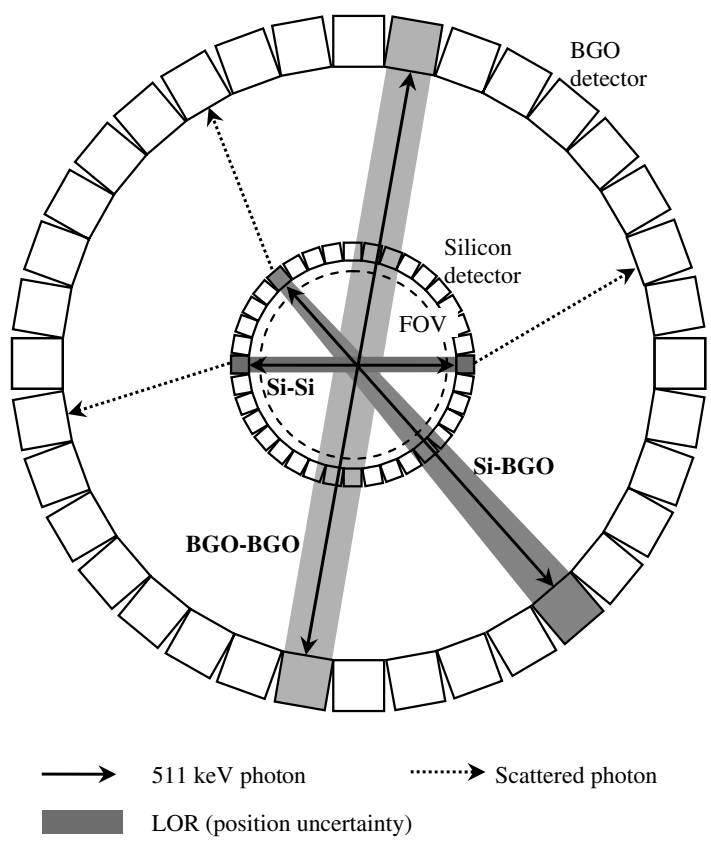

(b)

Figure 1. (a) A conceptual 3D drawing of a Compton PET device consisting of silicon pad detectors surrounded by position sensitive BGO detectors (not to scale). (b) Coincidence events: $\mathrm{Si}-\mathrm{Si}$ is a very high-resolution event. Si-BGO is a high-resolution event. BGO-BGO is a low-resolution event.

\section{Materials and methods}

\subsection{Concept of the proposed PET}

As an alternative for achieving sub-millimeter image resolution with good counting sensitivity, we previously proposed a very high-resolution PET concept based on high-spatial-resolution detectors surrounded by high-sensitivity scintillation detectors (Clinthorne et al 2000, Park et al 2001a, 2001b). More recently, similar concepts have been investigated by researchers (Tai et al 2003, Janecek et al 2006). Figure 1(a) shows a conceptual 3D drawing of a very high-resolution PET device. As shown in figure 1(a), the basic detector consists of an inner ring constructed of a material designed to Compton scatter $511 \mathrm{keV}$ photons and an outer ring comprised of a material to absorb either scattered or direct annihilation photons. Although the tomograph can be constructed using a variety of materials including scintillators in both the inner and outer rings, for this investigation solid-state silicon detectors were examined for the inner ring while the outer ring consisted of a more conventional BGO PET detector. The rationale for this choice is presented in section 2.2.

Referring to figure 1(b), each of the $511 \mathrm{keV}$ annihilation photons may undergo a number of interaction sequences in which the full energy is deposited in the detector system. The two most likely sequences are a single Compton scatter in the solid-state detector followed by absorption in the scintillation detector and absorption in the scintillation detector with no interaction in the solid-state detector. The various coincidence events of interest are $\mathrm{Si}-\mathrm{Si}$, 
Si-BGO and BGO-BGO where the silicon and BGO are used as the solid-state and scintillation detectors, respectively.

2.1.1. Si-Si interaction. A Si-Si coincidence event is an interaction in which each of the annihilation photons undergoes a single Compton scatter in the silicon detector followed by full absorption in the BGO detector. Si-Si interactions provide the highest spatial resolution since the single scattering event can be localized within the Compton recoil electron range if the pad size is sufficiently small. Moreover, the DOI uncertainty can be virtually eliminated by using multiple thin layers of silicon detectors. Note that the spatial resolution of these events is independent of the resolution of the second detector. Among the interactions of interest, $\mathrm{Si}-\mathrm{Si}$ events have the lowest probability.

2.1.2. Si-BGO interaction. In this interaction sequence, one photon of an annihilation pair undergoes a single Compton scatter in the silicon detector followed by full absorption in the BGO detector. The other photon penetrates the silicon detector with no interaction and is absorbed by the BGO detector. This process is defined as a Si-BGO interaction. Si-BGO events give intermediate spatial resolution since the energy is deposited over a larger volume in BGO, but the other end of the LOR is accurately determined in the silicon pad. Sensitivity midway between that for $\mathrm{Si}-\mathrm{Si}$ and BGO-BGO events is typically observed for the $\mathrm{Si}-\mathrm{BGO}$ coincidence because of the combination of the relatively lower probability of single scattering in the silicon detector and the high attenuation of the BGO detector. Even though the spatial resolution of $\mathrm{Si}-\mathrm{BGO}$ events depends on the position resolution of the second detector, the effect of the BGO resolution is demagnified in the small FOV. Moreover, the effect of poorer resolution in the second detector can be compensated by increasing its diameter relative to that of the first detector.

2.1.3. BGO-BGO interaction. A BGO-BGO coincidence is an interaction in which each of the annihilation photons undergoes penetration of the silicon detector with no interaction, followed by full absorption in the BGO detector. The BGO-BGO interaction is the conventional PET coincidence event and gives the lowest spatial resolution but the highest sensitivity due to the high attenuation of the BGO resulting both from photoelectric events and from Compton scatters followed by photoelectric absorption, as compared to the interaction probability in silicon.

Total energy is measured from the energy sum of both silicon and BGO detectors. Although the low energy resolution of BGO restricts the total energy resolution, the energy sum still can be used to reject annihilation photons that have scattered through large angles in the object.

\subsection{Initial analysis}

Monte Carlo simulations using the SKEPTIC code (Wilderman 1990) were performed to design a very high-resolution small animal PET instrument using a high-resolution solid-state scatter detector ring inserted into a conventional scintillator-based PET ring. The SKEPTIC code was developed at the University of Michigan for Monte Carlo simulations of photon transport in arbitrary 3D geometries. Historically, SKEPTIC has been more accurate than EGS4 below a few hundred $\mathrm{keV}$ due to its more accurate low-energy photon transport physics that also includes the Doppler broadening effect. Nevertheless, simulation results from current versions of EGS4 and GEANT4 agree well with the initial simulations conducted with SKEPTIC, and EGS4 was used for the follow-on simulations described in sections 2.3 and 2.4. 
Table 1. Fractions of interaction types and linear attenuation coefficients of detector materials for annihilation photons from the NIST XCOM database.

\begin{tabular}{llllll}
\hline $\begin{array}{l}\text { Detector } \\
\text { material }\end{array}$ & $\begin{array}{l}\text { Effective } \\
\text { atomic number }\end{array}$ & \% Coherent & \% Compton & \% Photoelectric & $\begin{array}{l}\text { Attenuation } \\
\left(\mathrm{cm}^{-1}\right)\end{array}$ \\
\hline BGO & 75 & 6 & 53 & 41 & 0.97 \\
LSO & 66 & 6 & 61 & 33 & 0.88 \\
NaI & 51 & 5 & 77 & 18 & 0.34 \\
Si & 14 & $<1$ & $\gg 99$ & $\ll 1$ & 0.20 \\
\hline
\end{tabular}

Table 1 shows the fraction of different interaction types for the first interaction of a $511 \mathrm{keV}$ photon and linear attenuation coefficients of PET detector materials from the National Institute of Standards and Technology (NIST) XCOM database. To simplify the event classification process in practice, it is highly desirable that the inner detector ring has a high probability of a Compton interaction followed by an escape of the scattered photon. Of the materials listed, silicon is the most appropriate for this task having more than $99 \%$ Compton interactions and almost no coherent or photoelectric interactions. BGO is well known as a very efficient gamma ray absorber due to the high atomic number of bismuth (83) and its high density $\left(7.13 \mathrm{~g} \mathrm{~cm}^{-3}\right)$. Even though timing and energy resolution will be lost by using BGO compared with LSO, BGO has intrinsically better performance in position estimation accuracy due to higher stopping power, lower inter-crystal scatter and less DOI uncertainty (Shao et al 1996, Park et al 2003). In addition, the production cost of BGO is lower than LSO. (Of course, as noted previously a variety of materials can be used to construct the inner and outer rings. Extensive investigations of optimum materials have not yet been conducted.)

The candidate design of a small animal PET consists of concentric cylindrical geometry with a silicon first detector surrounded by a BGO second detector. The size of the silicon detector was fixed with $4 \mathrm{~cm}$ ID and $4 \mathrm{~cm}$ length to fit a mouse. For this preliminary assessment of the proposed PET system, we did not include details such as animal beds, anesthetization equipment, system construction material, etc for the design parameters in this study. To accommodate larger animals, the diameters can be scaled up at the expense of sensitivity and a tolerable loss of resolution due to increased effects from acollinearity. There is no uniform optimum with respect to species, but a complex tradeoff between performance and cost. If maximum resolution is desired for the whole body, the animal will need to be translated axially. Increasing the length of the silicon detector increases efficiency but at increased cost.

The effects of Compton scattering, coherent scattering, penetration, absorption and Doppler broadening were included in simulations. In contrast to simulations in section 2.3, we assumed perfect energy resolution in the silicon and BGO detector. A narrow energy window $(1022 \pm 2 \mathrm{keV})$ was used. A total of 50000 photon pairs directed into the solid angle subtended by the silicon detector for each detector configuration were sampled from an isotropic point source located at the center of the FOV. Then the fractional solid angle (geometric sensitivity) was multiplied to the sensitivities measured (intrinsic sensitivities). A 3D Monte Carlo analysis was performed to examine the effect of various detector configurations (thickness, length and diameter) on the sensitivities of three major coincidence events ( $\mathrm{Si}-\mathrm{Si}, \mathrm{Si}-\mathrm{BGO}$ and BGO-BGO).

2.2.1. Thickness of the silicon detector. As silicon thickness increases, both single and multiple scattering events increase. The desirable events are those in which the gamma ray undergoes a single scatter followed by escape from the silicon and detection in the BGO. 
In order to estimate the effect of silicon detector thickness on sensitivity, simulations were performed with silicon thicknesses of $0.03,0.1,0.2,0.4,0.8,1.6,3.2$ and $6.4 \mathrm{~cm}$. The dimensions of the $\mathrm{BGO}$ detector were fixed at $24 \mathrm{~cm}$ diameter, $16 \mathrm{~cm}$ length and $2 \mathrm{~cm}$ thickness.

2.2.2. Thickness of the BGO detector. The probability of capture for a scattered photon from the silicon detector increases with the BGO detector thickness and finally saturates. The effect of BGO thickness was investigated with simulations using BGO detectors with $11.4 \mathrm{~cm}$ diameter, $16 \mathrm{~cm}$ length and various thicknesses $(0.1,0.2,0.4,0.8,1.0,1.5,2.0,4.0,8.0$ and $16 \mathrm{~cm})$. The silicon detector thickness was fixed at $1.6 \mathrm{~cm}$.

2.2.3. Diameter of the BGO detector. As the BGO diameter varies, one must consider the effects of changing geometry between the BGO and silicon resolution elements, acollinearity of annihilation photon pairs, DOI uncertainty on spatial resolution and the effect of changes in solid angle on sensitivity.

Changing the BGO diameter does not affect the resolution of $\mathrm{Si}-\mathrm{Si}$ events since it is determined only by the silicon ring diameter. Geometric resolution for $\mathrm{Si}-\mathrm{BGO}$ events improves as the BGO diameter increases (see figure 1(b)). At larger diameters, object resolution is more influenced by the high-resolution silicon interaction. Increasing the outer ring diameter does not change the geometric resolution for BGO-BGO coincidences, of course; however, annihilation photon acollinearity becomes a contributing factor.

For BGO-BGO (and $\mathrm{Si}-\mathrm{Si}$ ) events, the maximum image resolution loss from acollinearity occurs at the center of the FOV with the contribution to the width of the point spread function (PSF) well described by $0.0022 \times$ ring diameter (in the same distance measure). For $\mathrm{Si}-\mathrm{Si}$ events, the contribution $(0.09-0.16 \mathrm{~mm})$ is negligible due to the small ring diameter (from $40 \mathrm{~mm}$ at the innermost ring to $72 \mathrm{~mm}$ at the outermost ring). For a $17.6 \mathrm{~cm}$ diameter BGO ring it is $0.39 \mathrm{~mm}$ - also negligible because it is added in quadrature to the BGO detector responses. Interestingly, the effect on $\mathrm{Si}-\mathrm{BGO}$ resolution is both small and insensitive to the diameter of the outer ring. Specifically, if $R_{1}$ represents the distance from the annihilation to the silicon detector, $R_{2}$ the distance to the BGO detector and $\delta=0.0088 \mathrm{rad}$ FWHM angular uncertainty, the contribution to image resolution is given by $R_{1} R_{2} \delta\left(R_{1}+R_{2}\right)^{-1}$. This reduces to the conventional formula for the BGO-BGO and $\mathrm{Si}-\mathrm{Si}$ events where the maximum contribution is at the center. For the $\mathrm{Si}-\mathrm{BGO}$ events, however, the maximum resolution loss due to acollinearity is at the edge of the FOV, and the loss is relatively insensitive to the diameter of the outer ring. For example for $R_{1}=40 \mathrm{~mm}$, as $R_{2}$ increases from $88 \mathrm{~mm}$ to $500 \mathrm{~mm}$, the contribution due to acollinearity increases from $0.24 \mathrm{~mm}$ to only $0.32 \mathrm{~mm}$ FWHM.

Increasing the BGO ring diameter reduces the effect of DOI uncertainty for both BGO$\mathrm{BGO}$ and $\mathrm{Si}-\mathrm{BGO}$ coincidence events.

To examine the effect of BGO detector diameter on sensitivity, BGO diameters of 11.4, 14.0, 17.6, 23.0, 32.0 and $50.0 \mathrm{~cm}$ were simulated using a silicon ring detector consisting of $300 \mu \mathrm{m} \times 300 \mu \mathrm{m} \times 1 \mathrm{~mm}$ silicon pads and a BGO detector consisting of $3 \mathrm{~mm} \times 3 \mathrm{~mm} \times$ $20 \mathrm{~mm}$ BGO pixels. The diameter of the silicon detector was fixed (ID $4.0 \mathrm{~cm}$, OD $7.2 \mathrm{~cm}$ ).

In order to investigate the geometric resolution of Si-BGO events as a function of BGO diameter, we determined $\mathrm{Si}-\mathrm{BGO}$ resolution for the above diameters. Theoretically, the response function or coincidence sensitivity between a pair of detector elements in any given direction is maximum at the center and falls off to zero at the edge of the detector element. This factor is modeled as a triangular response function for Si-Si and BGO-BGO FWHM equal to one half the detector element width in both transaxial and axial directions at the center of 
the FOV. However, the profile of a Si-BGO event is trapezoidal-the coincidence sensitivity is maximum from the center to the edge of the silicon element and falls off to zero at the LOR connecting the edges of the silicon and the BGO element—since the joint solid angle is subtended by two different-sized opposing detector elements $(300 \mu \mathrm{m}$ silicon pixel and $3 \mathrm{~mm}$ BGO crystal) with the different radii of silicon $(2 \mathrm{~cm})$ and BGO $(5.7-25 \mathrm{~cm})$ scanners. The FWHMs were directly measured from the trapezoidal response functions.

2.2.4. Length of the BGO detector. As the length of the BGO cylinder is increased, the BGO solid angle subtended at the silicon detector increases to give increased sensitivity. In order to test the effect of the BGO detector length, various lengths of the BGO $(4,8,12$, and $16 \mathrm{~cm}$ ) cylinder for the second detector were simulated with a fixed diameter of $24 \mathrm{~cm}$ and thickness of $2 \mathrm{~cm}$. The silicon detector was used with a fixed $4 \mathrm{~cm}$ diameter, $4 \mathrm{~cm}$ length and $1.6 \mathrm{~cm}$ thickness.

\subsection{Evaluation of the proposed system}

A general-purpose package for Monte Carlo simulation, Electron-Gamma Shower code (EGS4), is used for the coupled transport of electrons and photons in 3D (Nelson et al 1985, Bielajew and Rogers 1987). Monte Carlo simulations using EGS4 were performed to evaluate the proposed PET system for very high-resolution animal imaging and demonstrate the sub-millimeter spatial resolution performance.

On the basis of the initial Monte Carlo studies described in section 2.2, a candidate PET design for small animals was chosen. It consists of two detector rings, a silicon detector (4 $\mathrm{cm}$ diameter, $4 \mathrm{~cm}$ length, and $1.6 \mathrm{~cm}$ thickness segmented into 16 layers of $300 \mu \mathrm{m} \times 300 \mu \mathrm{m} \times 1 \mathrm{~mm}$ pads $)$ and a BGO detector $(17.6 \mathrm{~cm}$ diameter, $16 \mathrm{~cm}$ length and $2 \mathrm{~cm}$ thickness segmented into $3 \mathrm{~mm} \times 3 \mathrm{~mm} \times 20 \mathrm{~mm}$ crystals). The energy resolution was simulated as 23\% FWHM at $511 \mathrm{keV}$ for BGO (Xie et al 2005). A constant resolution of $2.45 \mathrm{keV}$ at all energies for silicon detectors (Meier et al 2002) was used since the electronic noise contribution is dominant over the uncertainty in the number of electron-hole pairs generated in each interaction. A lower energy threshold (350 keV) was set to reject photons scattered in the object using the sum of silicon and BGO energies for each photon.

Due to inter-crystal scatter in the BGO detector, it is difficult to determine the first interaction position. Several methods have been investigated to estimate the detector element containing the first interaction (Comanor et al 1996, Shao et al 1996, Miyaoka and Lewellen 2000, Park et al 2003). These include the element with maximum energy, second maximum energy and the centroid of energy distribution in the scintillation detectors. Even though image resolution (FWHM and FWTM) is not significantly changed with different selection criteria, more events are assigned out of the correct LOR using the centroid method introducing significant noise and contrast loss in the image. Results indicate that selecting the crystal with the maximum or second maximum energy yields best performance depending on photon incidence angle (Miyaoka and Lewellen 2000). The maximum energy method is simplest to implement. In this and following studies the interaction position in the BGO detector was estimated by selecting the BGO element that has maximum energy deposited.

2.3.1. Spatial resolution and sensitivity. Simulations were performed with isotropic point sources in air to calculate spatial resolution and sensitivity for three major coincidence events ( $\mathrm{Si}-\mathrm{Si}, \mathrm{Si}-\mathrm{BGO}$ and BGO-BGO). A total of 10 million photon pairs were sampled. Point sources were located at the center of the FOV and at $0.3,0.6,0.9,1.21 .5$ and $1.8 \mathrm{~cm}$ radius at 
the axial center of the scanner. Data were also acquired from on-axis sources at 0.0, 0.3, 0.6, $0.9,1.21 .5$ and $1.8 \mathrm{~cm}$ from the axial center.

In order to define the effect of detector element size on the intrinsic scanner resolution, effects of acollinearity and blurring due to positron range were not considered in this simulation. To eliminate the need for developing an appropriate volume PET reconstruction at this stage of the research, the recorded 3D event data were projected axially onto a $2 \mathrm{D}$ virtual source plane and $540 \times 840$ (distance $\times$ angle) sinograms were histogrammed. Each sinogram bin was $0.074 \mathrm{~mm}$ by $0.214^{\circ}$. Images were reconstructed using $2 \mathrm{D}$ FBP with a ramp filter having a cutoff frequency of $6.8 \mathrm{~mm}^{-1}$. Reconstructed images closely represent the line response rather than the point response of the tomograph using this $3 \mathrm{D}$ to $2 \mathrm{D}$ 'squashing' method.

2.3.2. Annihilation photon acollinearity. Since the center of mass of the positron and electron is in motion when the positron and electron annihilate, the two $511 \mathrm{keV}$ annihilation photons are emitted with an uncertainty in the $180^{\circ}$ angle between them in order to conserve energy and momentum. A Gaussian distribution with $0^{\circ}$ mean and $0.5^{\circ}$ FWHM $(0.0088 \mathrm{rad})$ is used to sample angular uncertainty presented by Evans (1995) and Harrison et al (1999). The direction vector of one photon of the annihilation pair is randomly sampled. The direction vector of the other photon is opposite to the first, but is deflected with the uncertainty in polar angle and a randomly sampled azimuthal angle.

2.3.3. Positron range. In positron decay, one of the protons in the nucleus is transformed into a neutron. A positron is emitted with a continuous energy distribution up to a maximum energy since it shares transformation energy with a neutrino. A positron travels with the kinetic energy from the decay position until it annihilates with an electron. The positron range degrades resolution as a result of the uncertainty in the position of the positron emission. Derenzo (1979, 1986) and Haber et al (1990) measured positron range distribution from experiments and proposed positron range blurring functions. Palmer and Brownell (1992) developed an evaluation model with positron energy distribution and an empirical positron range formula. Levin and Hoffman (1999) exploited a Monte Carlo code that simulates positron trajectories and presented the spatial uncertainty (FWHM and FWTM directly measured from cusp-like distribution) from positron range and the positron energy for isotopes. Because of the cusp-like distribution of the positron range with long tails, the distribution is not well described by the Gaussian function. Therefore, the FWHM is not the best indicator of the effect of the positron range. A distribution function derived by fitting the data from simulations is

$$
P(x)=C \mathrm{e}^{-k_{1} x}+(1-C) \mathrm{e}^{-k_{2} x},
$$

where $C$ is $0.516, k_{1}$ is $37.9 \mathrm{~mm}^{-1}$, and $k_{2}$ is $3.10 \mathrm{~mm}^{-1}$ for the ${ }^{18} \mathrm{~F}$ isotope (Levin and Hoffman 1999). Since the direct positron tracking method using Monte Carlo simulation is too computationally intensive, a sinogram blurring method was used to model positron range effects. The cusp-like positron range distribution of the ${ }^{18} \mathrm{~F}$ isotope from equation (1) was convolved with the sinogram space.

2.3.4. Recoil electron range. Similar to multiple gamma ray scattering in the scintillation detector, migration of a recoil electron between adjacent pads of the silicon detector can degrade the spatial resolution due to misplacement of the gamma ray interaction position. Therefore, the most serious intrinsic resolution limit for the proposed instrument is imposed by the range of the Compton recoil electron. Moreover, the recoil electron can escape the 


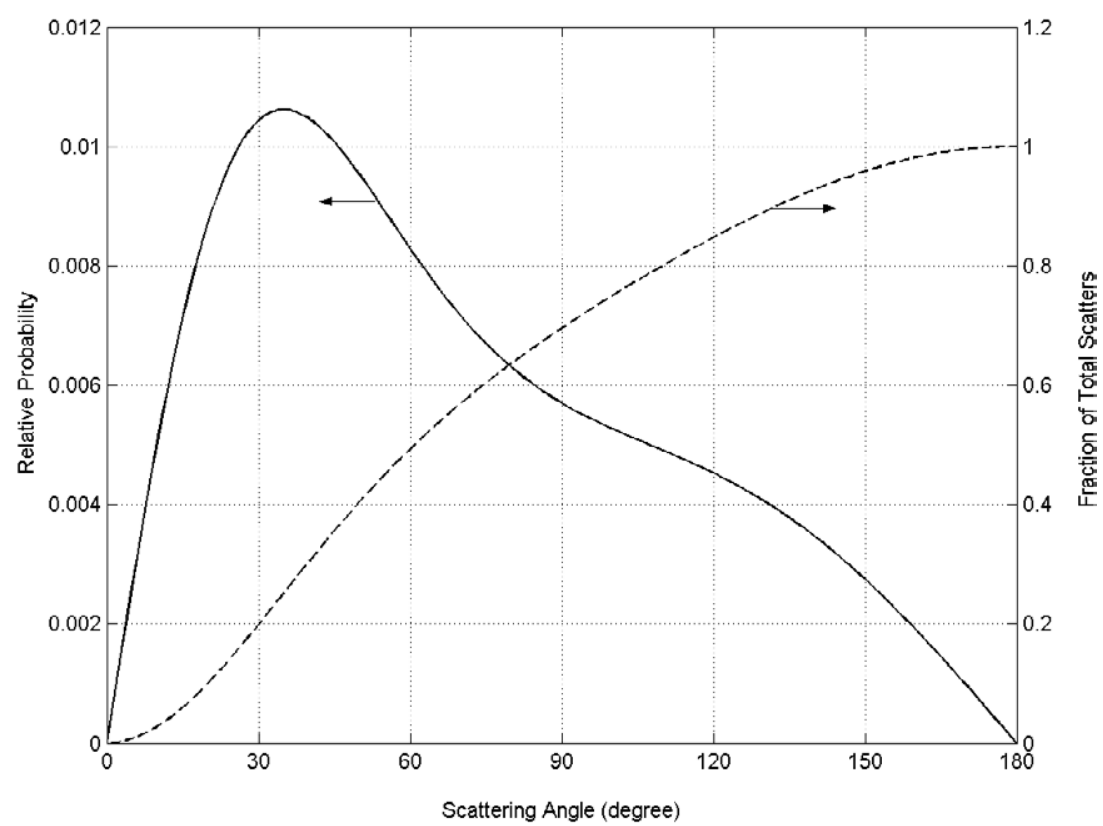

Figure 2. Probability of Compton scattering and the fraction of the total scatters in silicon for $511 \mathrm{keV}$ photons.

detector without depositing its entire energy. Even though this energy loss can reduce the total energy sum used to reject scatter events in the object, it will typically not be significant since the energy uncertainty of the BGO detector is dominant.

Figure 2 shows the probability of angular distribution for Compton-scattered gamma rays and the fraction of the total scatters in silicon for $511 \mathrm{keV}$ photons predicted by the KleinNishina formula (Knoll 1999 p 51). The most probable angle of scatter is about $35^{\circ}$. The scatter fraction at angles less than $90^{\circ}$ is $70 \%$ of total scatter.

Recoil electrons may lose energy by radiative processes as well as by Coulomb interactions. The ESTAR program of the NIST is used to estimate the recoil electron range in silicon. The kinetic energy of the recoil electron, $E_{\text {recoil }}$, is

$$
E_{\text {recoil }}=E_{\text {photon }} \frac{(1-\cos \theta)}{(2-\cos \theta)},
$$

where $E_{\text {photon }}$ is $511 \mathrm{keV}$ and $\theta$ is the scattering angle of the photon. Table 2 shows the continuous slowing down approximation (CSDA) range in silicon for various electron energies. In addition to figure 2 and table 2, a previous EGS4 Monte Carlo simulation study (Park et al 2002), in which electron paths were tracked within the silicon, has shown that the overall effect of recoil electron range on spatial resolution is not significant for the detector element size used here. Recoil electron range was therefore not considered in the present investigation.

2.3.5. Overall resolution and image characteristics. In order to investigate overall resolution and image quality of the proposed scanner design, simulations were performed with distributed sources. The effects of positron range and annihilation photon acollinearity were included in the both simulations. The simulation and image reconstruction method for overall resolution are the same as in section 2.3.1. 
Table 2. Continuous slowing down approximation range in silicon for various electron energies from the NIST ESTAR database.

\begin{tabular}{llll}
\hline $\begin{array}{l}\gamma \text { scattering } \\
\text { angle }\left(^{\circ}\right)\end{array}$ & $\begin{array}{l}\text { Recoil electron } \\
\text { energy }(\mathrm{keV})\end{array}$ & $\begin{array}{l}\text { Range } \times \text { density } \\
\left(\mathrm{g} \mathrm{cm}^{-2}\right)\end{array}$ & $\begin{array}{l}\text { CSDA } \\
\text { range }(\mu \mathrm{m})\end{array}$ \\
\hline 30 & 60 & $7.65 \times 10^{-3}$ & 33 \\
60 & 171 & $4.40 \times 10^{-2}$ & 189 \\
90 & 256 & $8.74 \times 10^{-2}$ & 375 \\
120 & 307 & $1.09 \times 10^{-1}$ & 468 \\
150 & 333 & $1.23 \times 10^{-1}$ & 528 \\
180 & 340 & $1.27 \times 10^{-1}$ & 544 \\
\hline
\end{tabular}

To evaluate image quality of the candidate design, a simulation was performed with a multiple cylinder source. The simulated source consisted of six cylinders. The center of each was located at $1 \mathrm{~cm}$ from the center of the FOV. Cylinder diameters were $0.1,0.2,0.4,0.6,0.8$ and $1.0 \mathrm{~cm}$ with $1.0 \mathrm{~cm}$ height. A total of 20 million photon pairs were uniformly sampled in the six cylinders based on source volume. The sources emitted photons isotropically. For the image reconstruction of the multiple cylinder source, a ramp filter with a Hamming window having a cutoff frequency of $3.4 \mathrm{~mm}^{-1}$ was used to reduce high-frequency noise amplification.

The distributed point sources and the multiple cylinder source were embedded into a larger $4.0 \mathrm{~cm}$ diameter $\times 4.0 \mathrm{~cm}$ long cylinder containing water to consider scattering in the object. A portion of the scatter was rejected using a lower energy threshold $(350 \mathrm{keV})$ on the measured energy. While scatter events in the object and detectors were included, random coincidences and deadtime effects, which depend strongly on count rate, timing resolution and design of system electronics, were not included in this study.

\subsection{Timing simulation}

The noise equivalent count rate (NECR) performance of the proposed PET was investigated with Monte Carlo simulation data and timing simulations. The interaction information including interaction type, exact interaction position in 3D and deposited energy in detectors was used from the previous Monte Carlo simulation data in section 2.3.5 using a point source located at the radial and axial center of the PET in the $4.0 \mathrm{~cm}$ diameter $\times 4.0 \mathrm{~cm}$ long water cylinder.

Interaction timing information was estimated with decay interval, time of flight (TOF) and the timing uncertainty of the silicon and BGO detectors. Decay time intervals of an annihilation photon source were generated assuming a homogenous Poisson point process for various source activities $(0.1,0.3,0.5,0.7,1.0,1.5,2,3$ and $5 \mathrm{mCi})$. The TOF between the first interaction position and the origin of photon was calculated with the distance between two positions and the speed of light $\left(3 \times 10^{8} \mathrm{~m} \mathrm{~s}^{-1}\right)$ used for the speed of the photons. In the case of multiple scatters in a detector, the TOFs between the scatter positions were calculated based on the distances between interaction positions in 3D with proper interaction sequence. Simulation includes the time delay caused from scatters in the object even though this delay is negligibly small due to the small system diameter. The timing uncertainty of the silicon detector was assumed to have a Gaussian distribution (5 ns FWHM for single events and $7 \mathrm{~ns}$ FWHM for coincidence events) since the triggering uncertainty is governed primarily by electronic noise and the timing resolution is achievable using time-walk compensation. For the timing of the BGO coupled to a photomultiplier, a mono-exponential BGO scintillation 


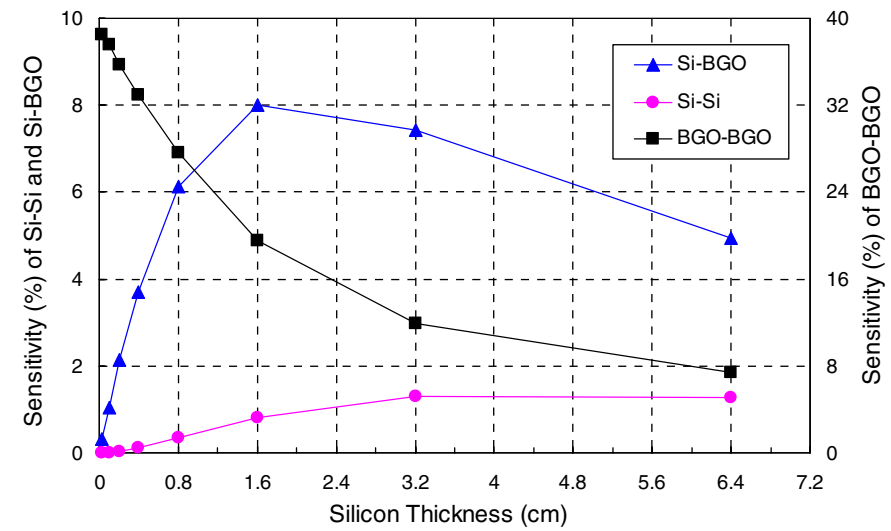

Figure 3. Plot of sensitivities of $\mathrm{Si}-\mathrm{Si}$, Si-BGO and BGO-BGO events as a function of silicon thickness, using a $4 \mathrm{~cm}$ diameter and $4 \mathrm{~cm}$ length silicon detector surrounded by a $24 \mathrm{~cm}$ diameter, $16 \mathrm{~cm}$ length and $2 \mathrm{~cm}$ thick BGO detector.

model having a time constant of $300 \mathrm{~ns}$ and 300 photoelectron yield at $511 \mathrm{keV}$ was used. The effect of the system deadtime was not included in this preliminary NECR study since deadtime is very implementation-specific and its effects on such a system are extremely complex. An energy window of 511-1533 keV for total energy sum and $7 \mathrm{~ns}$ timing window were used to measure coincidence events.

The NECR was calculated using the following formula:

$$
\mathrm{NECR}=\frac{T^{2}}{T+S+R+M},
$$

where $T, S, R$ and $M$ denote the (assumed known) rates of detected true, scattered, random coincidence and misclassified events, respectively. A misclassified event is defined as a coincidence having event loss in the silicon detector due to the timing uncertainty of the silicon detector. Since it is generated from the same decay event, it is distinct from random events generated from the different positron decays. For example, a Si-Si event can be misclassified into a $\mathrm{Si}-\mathrm{BGO}$ event due to an event loss in one silicon detector or a BGO-BGO event resulting from both interactions loss in the silicon detector pair. Coincidence events resulting in an LOR lying outside the $4 \mathrm{~cm}$ FOV were rejected before NECR calculation.

\section{Results}

\subsection{Initial analysis}

The effect of silicon thickness on the detection sensitivity of coincidence events ( $\mathrm{Si}-\mathrm{Si}, \mathrm{Si}-$ $\mathrm{BGO}$ and BGO-BGO) is shown in figure 3. Maximum sensitivity (8.0\%) for Si-BGO events was obtained with $1.6 \mathrm{~cm}$ silicon thickness. The sensitivity of this event class then decreases since the probability for photons penetrating the silicon detector and interaction with the BGO decreases with increasing silicon thickness. Multiple scattering events in the silicon detector, which are rejected, also increase with silicon thickness. $\mathrm{Si}-\mathrm{Si}$ events have a maximum sensitivity of $1.3 \%$ at about $3.2 \mathrm{~cm}$ silicon thickness and decrease slowly due to increased multiple scattering in thicker silicon. BGO-BGO events decrease exponentially from $38.5 \%$ to $7.3 \%$ as silicon thickness increases from $0.03 \mathrm{~cm}$ to $6.4 \mathrm{~cm}$. 


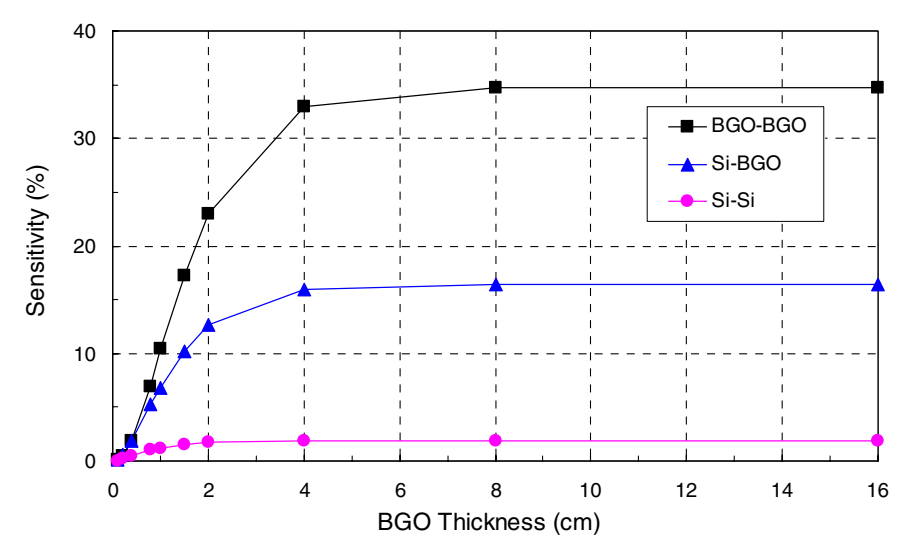

Figure 4. Plot of sensitivities of $\mathrm{Si}-\mathrm{Si}, \mathrm{Si}-\mathrm{BGO}$ and BGO-BGO events as a function of BGO thickness, using an $11.4 \mathrm{~cm}$ diameter and $16 \mathrm{~cm}$ length $\mathrm{BGO}$ detector surrounding a $4 \mathrm{~cm}$ diameter, $4 \mathrm{~cm}$ length and $1.6 \mathrm{~cm}$ thick silicon detector.

Figure 4 shows the sensitivities of the coincidence events ( $\mathrm{Si}-\mathrm{Si}, \mathrm{Si}-\mathrm{BGO}$ and BGO$\mathrm{BGO})$ as a function of BGO thickness. Because the Si detector ring is completely inside the $\mathrm{BGO}$ detector ring, one may expect that the sensitivity of the $\mathrm{Si}-\mathrm{Si}$ event will not change as a function of BGO thickness. However, not only the sensitivities of the $\mathrm{Si}$ BGO and BGO-BGO coincidence events but also the sensitivity of the $\mathrm{Si}-\mathrm{Si}$ event varies as a function of BGO thickness. As described with the definition of the Si-Si coincidence event in section 2.1.1, the $\mathrm{Si}-\mathrm{Si}$ coincidence event consists of two processes-a single Compton scatter in the silicon detector and full absorption in the BGO detector. Since the energy range of the photons scattered from the silicon detector is $171-511 \mathrm{keV}$, the scattered photons still can penetrate the BGO detector without interaction. Therefore, the sensitivity of the Si-Si event is dependent on the BGO thickness. Sensitivities of all three event types are saturated for 4 $\mathrm{cm}$ BGO. For a BGO thickness of $2 \mathrm{~cm}$, we obtained sensitivities of $90 \%$ for $\mathrm{Si}-\mathrm{Si}, 78.8 \%$ for $\mathrm{Si}-\mathrm{BGO}$ and $69.5 \%$ for BGO-BGO compared to that obtained with a $4 \mathrm{~cm}$ BGO thickness.

The effect of the BGO diameter on sensitivity is plotted in figure 5. Sensitivities of the three types of desirable coincidence events decline since the capture solid angle of the BGO detector decreases with increasing ring diameter for a fixed length. However, by increasing the BGO diameter from $11.4 \mathrm{~cm}$ to $50.0 \mathrm{~cm}$, the spatial resolution of Si-BGO events improves from about $0.65 \mathrm{~mm}$ to $0.4 \mathrm{~mm}$ FWHM at the cost of a decrease in sensitivity from $12.6 \%$ to $2.5 \%$. The Si-BGO spatial resolution is $0.65,0.6,0.55,0.5,0.45$ and $0.4 \mathrm{~mm} \mathrm{FWHM}$ at the center of the FOV for the six BGO diameters listed in section 2.2.3. Actual image resolution may be degraded due to the effect of positron range, acollinearity of annihilation photon pairs and inter-crystal scatter.

The sensitivity for coincidence events as a function of the BGO detector length is illustrated in figure 6. By increasing the $\mathrm{BGO}$ length by a factor of 4 , the sensitivities of $\mathrm{Si}-\mathrm{Si}, \mathrm{Si}-\mathrm{BGO}$, BGO-BGO increase by a factor of 13,11 and 4 , respectively.

Based on the initial analysis, a candidate instrument was designed with a $4 \mathrm{~cm}$ diameter, $4 \mathrm{~cm}$ length and $1.6 \mathrm{~cm}$ thick silicon cylinder surrounded by a $17.6 \mathrm{~cm}$ diameter, $16 \mathrm{~cm}$ long and $2 \mathrm{~cm}$ thick BGO cylinder. The length and diameter of the silicon detector were designed to image mice. The silicon thickness was obtained to maximize the sum of very high-resolution $\mathrm{Si}-\mathrm{Si}$ events and high-resolution $\mathrm{Si}-\mathrm{BGO}$ events. The BGO diameter was picked to have 


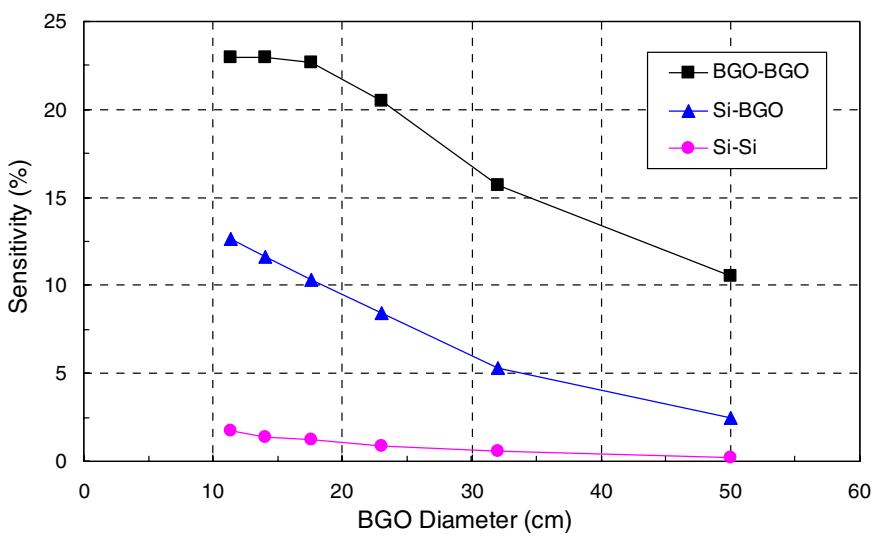

Figure 5. Plot of detection sensitivities of $\mathrm{Si}-\mathrm{Si}, \mathrm{Si}-\mathrm{BGO}$ and $\mathrm{BGO}-\mathrm{BGO}$ events as a function of BGO diameter, using a $16 \mathrm{~cm}$ length and $2 \mathrm{~cm}$ thickness BGO detector surrounding a $4 \mathrm{~cm}$ diameter, $4 \mathrm{~cm}$ length and $1.6 \mathrm{~cm}$ thick silicon detector.

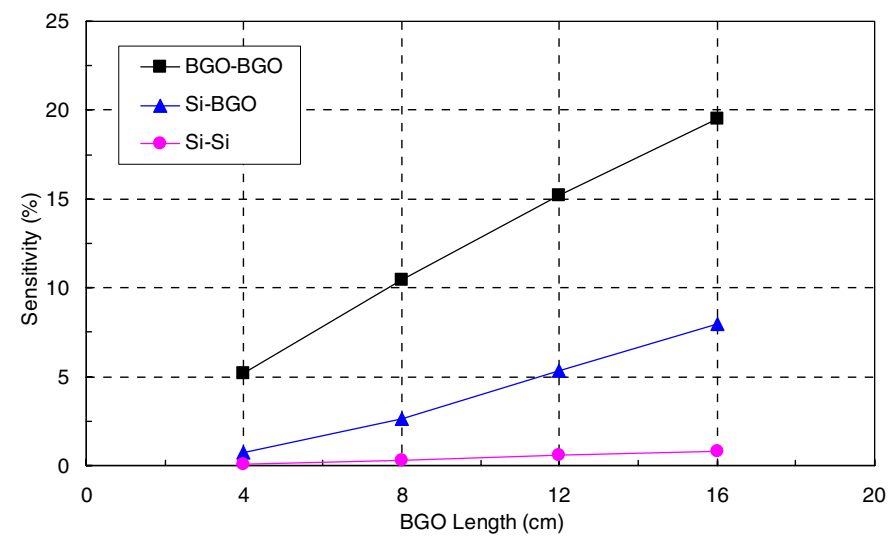

Figure 6. Plot of detection sensitivities of $\mathrm{Si}-\mathrm{Si}, \mathrm{Si}-\mathrm{BGO}$ and $\mathrm{BGO}-\mathrm{BGO}$ events as a function of BGO length, using a $24 \mathrm{~cm}$ diameter and $2 \mathrm{~cm}$ thickness BGO detector surrounding a $4 \mathrm{~cm}$ diameter, $4 \mathrm{~cm}$ length and $1.6 \mathrm{~cm}$ thick silicon detector.

geometric resolution of $0.55 \mathrm{~mm}$ FWHM at the center of the FOV for Si-BGO events using $300 \mu \mathrm{m} \times 300 \mu \mathrm{m}$ silicon pads and $3 \mathrm{~mm} \times 3 \mathrm{~mm}$ BGO crystals. This resolution does not include acollinearity for the Si-BGO events, which increases the overall width to $0.57-$ $0.60 \mathrm{~mm}$. The BGO thickness was chosen to match commercially available detectors. The BGO length was selected arbitrarily to obtain reasonable sensitivity for system evaluation.

\subsection{Evaluation of the proposed system}

The detection sensitivities with point sources located at various radial and axial offset positions are summarized in table 3. Sensitivities as a function of radial source position for the three event classes were uniformly distributed in the FOV. Sensitivity as a function of axial source position for $\mathrm{Si}-\mathrm{Si}$ events decreases to 0 at the edge of the silicon ring. However, sensitivity of Si-BGO events only decreases to $70 \%$ of that for zero axial offset demonstrating significant sensitivity for high-resolution events even beyond the $4 \mathrm{~cm}$ axial extent of the silicon. 
Table 3. Sensitivities of three different resolution events.

\begin{tabular}{|c|c|c|c|c|c|c|}
\hline \multirow{3}{*}{$\begin{array}{l}\text { Point source offset } \\
\text { position }(\mathrm{cm})\end{array}$} & \multicolumn{6}{|c|}{ Sensitivity (\%) at radial and axial source positions } \\
\hline & \multicolumn{2}{|c|}{$\mathrm{Si}-\mathrm{Si}$} & \multicolumn{2}{|c|}{$\mathrm{Si}-\mathrm{BGO}$} & \multicolumn{2}{|c|}{ BGO-BGO } \\
\hline & Radial & Axial & Radial & Axial & Radial & Axial \\
\hline 0.0 & 1.04 & 1.04 & 9.09 & 9.09 & 21.13 & 21.13 \\
\hline 0.3 & 1.03 & 0.98 & 9.08 & 8.99 & 21.01 & 21.07 \\
\hline 0.6 & 1.02 & 0.85 & 9.09 & 8.68 & 20.70 & 21.14 \\
\hline 0.9 & 1.02 & 0.71 & 9.11 & 8.21 & 20.30 & 21.45 \\
\hline 1.2 & 1.04 & 0.54 & 9.12 & 7.63 & 19.79 & 21.88 \\
\hline 1.5 & 1.09 & 0.35 & 9.15 & 6.99 & 19.15 & 22.29 \\
\hline 1.8 & 1.21 & 0.15 & 9.23 & 6.34 & 18.17 & 22.73 \\
\hline
\end{tabular}

Table 4. Spatial resolution (FWHM) of three different events versus the radial source position.

\begin{tabular}{|c|c|c|c|c|c|c|}
\hline \multirow{3}{*}{$\begin{array}{l}\text { Point source radial } \\
\text { offset position }(\mathrm{cm})\end{array}$} & \multicolumn{6}{|c|}{ Radial and tangential resolution (mm FWHM) } \\
\hline & \multicolumn{2}{|c|}{$\mathrm{Si}-\mathrm{Si}$} & \multicolumn{2}{|c|}{$\mathrm{Si}-\mathrm{BGO}$} & \multicolumn{2}{|c|}{ BGO-BGO } \\
\hline & Radial & Tangential & Radial & Tangential & Radial & Tangential \\
\hline 0.0 & 0.23 & 0.22 & 0.79 & 0.80 & 1.45 & 1.53 \\
\hline 0.3 & 0.24 & 0.24 & 0.80 & 0.78 & 2.44 & 2.49 \\
\hline 0.6 & 0.25 & 0.25 & 0.79 & 0.76 & 2.61 & 2.65 \\
\hline 0.9 & 0.26 & 0.26 & 0.75 & 0.69 & 2.85 & 2.13 \\
\hline 1.2 & 0.30 & 0.29 & 0.77 & 0.63 & 2.69 & 2.28 \\
\hline 1.5 & 0.34 & 0.33 & 0.74 & 0.55 & 2.69 & 2.36 \\
\hline 1.8 & 0.38 & 0.37 & 0.74 & 0.49 & 3.01 & 2.42 \\
\hline
\end{tabular}

Table 5. Spatial resolution (FWHM) of three different events versus the axial source position.

\begin{tabular}{|c|c|c|c|c|c|c|}
\hline \multirow{3}{*}{$\begin{array}{l}\text { Point source axial } \\
\text { offset position }(\mathrm{cm})\end{array}$} & \multicolumn{6}{|c|}{ Radial and tangential resolution (mm FWHM) } \\
\hline & \multicolumn{2}{|c|}{$\mathrm{Si}-\mathrm{Si}$} & \multicolumn{2}{|c|}{$\mathrm{Si}-\mathrm{BGO}$} & \multicolumn{2}{|c|}{ BGO-BGO } \\
\hline & Radial & Tangential & Radial & Tangential & Radial & Tangential \\
\hline 0.0 & 0.23 & 0.22 & 0.79 & 0.80 & 1.45 & 1.53 \\
\hline 0.3 & 0.23 & 0.22 & 0.76 & 0.78 & 1.49 & 1.52 \\
\hline 0.6 & 0.23 & 0.23 & 0.80 & 0.80 & 1.48 & 1.61 \\
\hline 0.9 & 0.23 & 0.23 & 0.80 & 0.79 & 1.53 & 1.47 \\
\hline 1.2 & 0.23 & 0.23 & 0.79 & 0.77 & 1.58 & 1.52 \\
\hline 1.5 & 0.23 & 0.23 & 0.80 & 0.82 & 1.62 & 1.50 \\
\hline 1.8 & 0.23 & 0.23 & 0.79 & 0.77 & 1.54 & 1.58 \\
\hline
\end{tabular}

Tables 4 and 5 show the radial and tangential image resolutions as a function of radial and axial source position, respectively. These spatial resolutions exclude the effects of positron range and annihilation photon acollinearity. Spatial resolutions were calculated using Gaussian fit of the PSF at the center of the FOV. The very high resolution, $220 \mu \mathrm{m}$ FWHM with $1.04 \%$ detection sensitivity was achieved from $\mathrm{Si}-\mathrm{Si}$ events. Si-BGO events gave high resolution, $790 \mu \mathrm{m}$ FWHM with $9.09 \%$ sensitivity. The conventional PET resolution, $1.45 \mathrm{~mm}$ FWHM with high sensitivity $21.13 \%$, was obtained from BGO-BGO events. For each event class, transaxial resolution as a function of the axial source position does not deteriorate even though the sensitivity of Si-Si significantly decreases. 


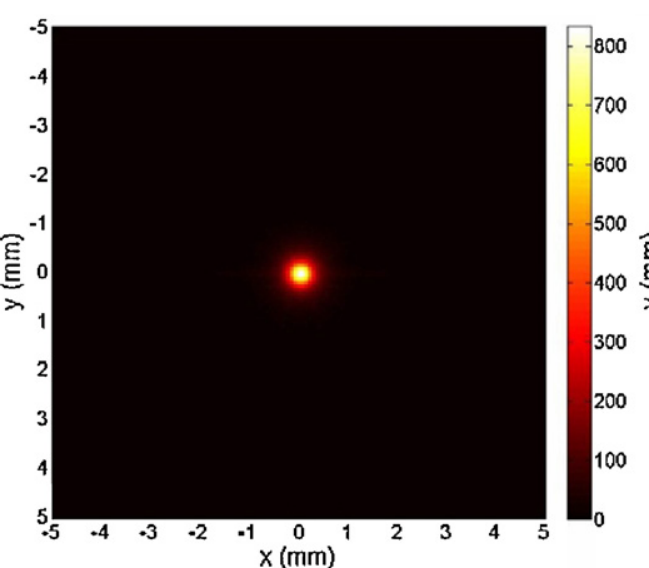

(a)

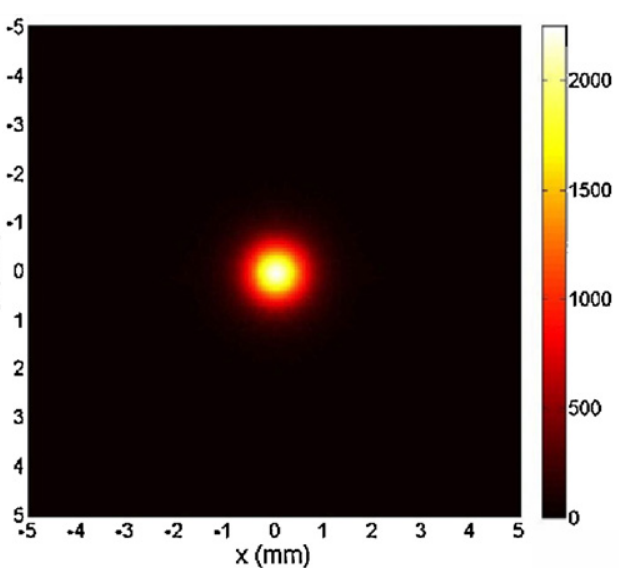

(b)

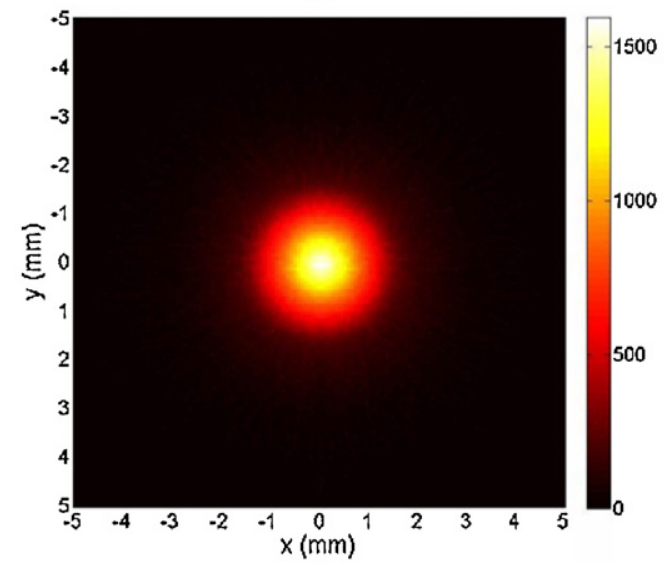

(c)

Figure 7. Images of a ${ }^{18} \mathrm{~F}$ point source in water from (a) $\mathrm{Si}-\mathrm{Si}$, (b) $\mathrm{Si}-\mathrm{BGO}$ and (c) $\mathrm{BGO}-\mathrm{BGO}$ coincidence events. All images include the effects of annihilation photon acollinearity, positron range of ${ }^{18} \mathrm{~F}$ and scatter.

The results of Monte Carlo simulation study including only annihilation photon acollinearity show that the spatial resolution is very slightly degraded to $240 \mu \mathrm{m}$ FWHM for $\mathrm{Si}-\mathrm{Si}, 820 \mu \mathrm{m}$ FWHM for Si-BGO and $1.46 \mathrm{~mm}$ FWHM for BGO-BGO events as we expected in section 2.3.2.

Figure 7 shows images of the ${ }^{18} \mathrm{~F}$ point source in water from $\mathrm{Si}-\mathrm{Si}, \mathrm{Si}-\mathrm{BGO}$ and BGOBGO coincidence events. Each image was normalized to an individual maximum. Each color bar to the right of each image shows counts in a pixel. 1D profiles of the images are shown in figure 8 . The profiles are normalized to total counts in a central slice of image for a point source at the center of the FOV. Tables 6 and 7 show the overall image resolutions as a function of radial and axial source position, respectively. Spatial resolutions were calculated using a Gaussian fit to the PSF except for $\mathrm{Si}-\mathrm{Si}$ events. As shown in figure 8, the PSF of $\mathrm{Si}-\mathrm{Si}$ events does not follow a Gaussian distribution but rather has a cusp-like distribution since the blurring from positron range dominates the PSF. The FWHM of Si-Si events in tables 6 and 7 were directly measured from the PSF. The overall resolution for $\mathrm{Si}-\mathrm{Si}, \mathrm{Si}-\mathrm{BGO}$ and BGO-BGO events is $340 \mu \mathrm{m}, 1.01 \mathrm{~mm}$ and $1.66 \mathrm{~mm}$ FWHM, respectively at the center of the 


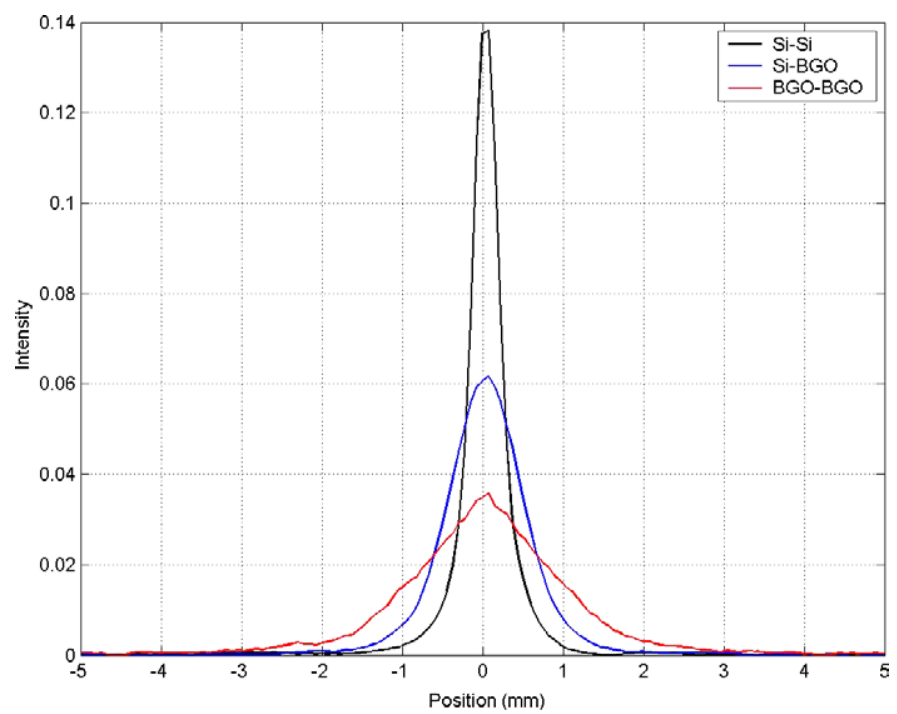

Figure 8. 1D profiles of ${ }^{18} \mathrm{~F}$ point source images reconstructed with $\mathrm{Si}-\mathrm{Si}$, Si-BGO and BGOBGO events. The point source located at the center of the FOV.

Table 6. Overall spatial resolution (FWHM) of three different events versus the radial source position.

\begin{tabular}{|c|c|c|c|c|c|c|}
\hline \multirow{3}{*}{$\begin{array}{l}\text { Point source radial } \\
\text { offset position }(\mathrm{cm})\end{array}$} & \multicolumn{6}{|c|}{ Radial and tangential resolution (mm FWHM) } \\
\hline & \multicolumn{2}{|c|}{$\mathrm{Si}-\mathrm{Si}$} & \multicolumn{2}{|c|}{$\mathrm{Si}-\mathrm{BGO}$} & \multicolumn{2}{|c|}{ BGO-BGO } \\
\hline & Radial & Tangential & Radial & Tangential & Radial & Tangential \\
\hline 0.0 & 0.37 & 0.34 & 1.01 & 1.01 & 1.73 & 1.66 \\
\hline 0.3 & 0.37 & 0.35 & 1.00 & 1.00 & 2.66 & 2.82 \\
\hline 0.6 & 0.38 & 0.36 & 0.99 & 0.97 & 2.76 & 2.73 \\
\hline 0.9 & 0.41 & 0.38 & 0.97 & 0.94 & 2.72 & 2.49 \\
\hline 1.2 & 0.44 & 0.42 & 0.95 & 0.90 & 2.79 & 2.58 \\
\hline 1.5 & 0.48 & 0.48 & 0.94 & 0.91 & 2.90 & 2.69 \\
\hline 1.8 & 0.51 & 0.58 & 0.91 & 0.87 & 3.03 & 2.68 \\
\hline
\end{tabular}

FOV. Although random coincidences and deadtime were ignored at this stage of our analysis, their inclusion, while it might have a significant effect on the NECR, will have little overall effect on spatial resolution.

Finally, 2D images in figure 9 were reconstructed with the three different resolution datasets. There is no attenuation correction in the image. The relative number of coincidence events (160k Si-Si events, 1.4 M Si-BGO events and 3 M BGO-BGO events) for each dataset based on the relative sensitivity was used for reconstructions. The system generates a very high-resolution (high noise) image from $\mathrm{Si}-\mathrm{Si}$ events and a low-resolution (low noise) image from BGO-BGO events.

\subsection{Timing simulation}

In figures 10-12, the results show that the rates of the scatter, random and misclassified events from the $\mathrm{Si}-\mathrm{Si}, \mathrm{Si}-\mathrm{BGO}$ and BGO-BGO events increase by increasing source activity. The 


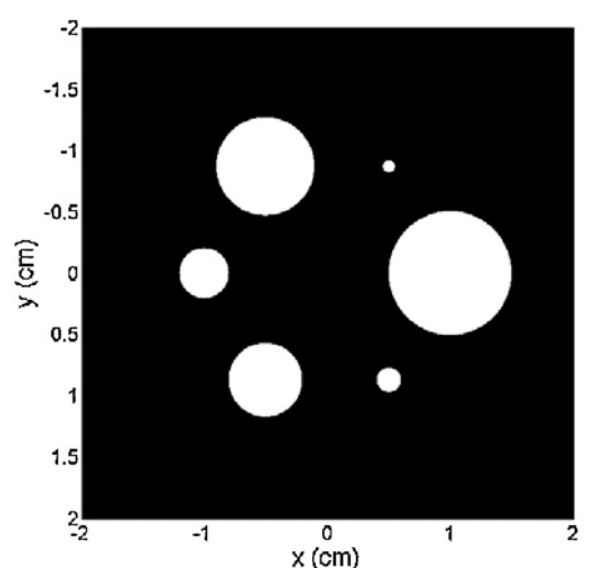

(a)

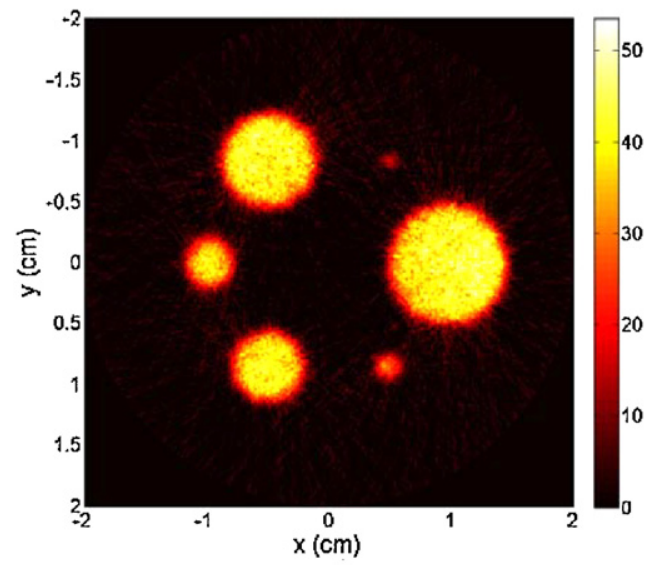

(c)

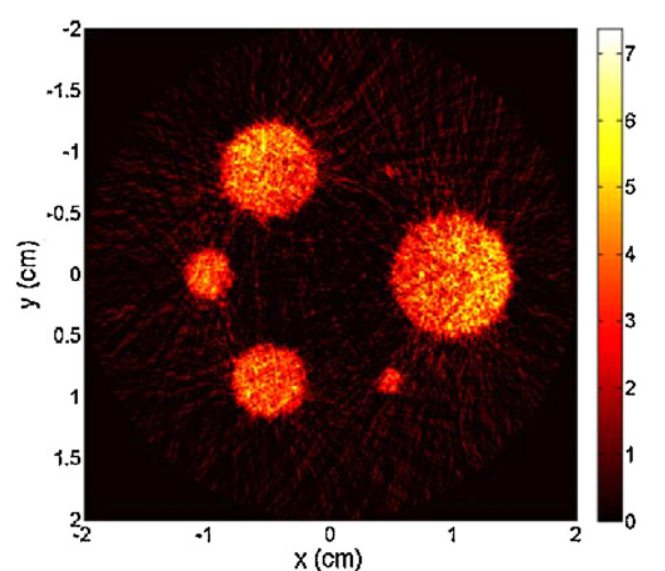

(b)

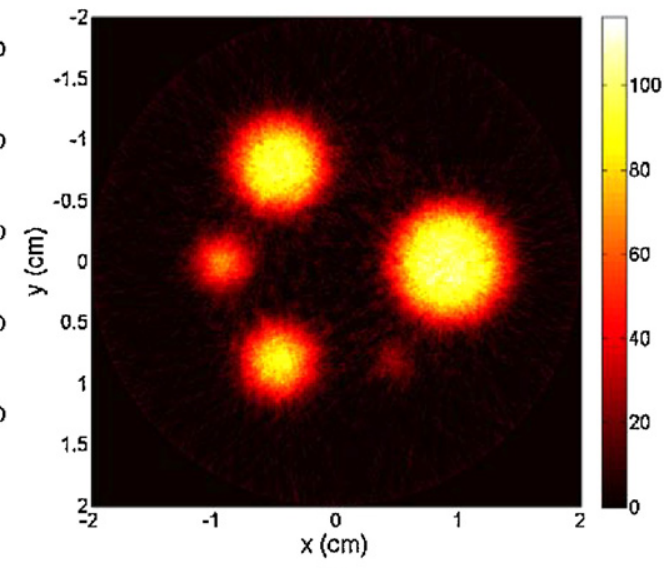

(d)

Figure 9. (a) $\mathrm{A}^{18} \mathrm{~F}$ multiple cylinder source in water and images from (b) $\mathrm{Si}-\mathrm{Si}$, (c) $\mathrm{Si}-\mathrm{BGO}$ and (d) BGO-BGO coincidence events. All images include the effects of annihilation photon acollinearity, positron range of ${ }^{18} \mathrm{~F}$ and scatter.

Table 7. Overall spatial resolution (FWHM) of three different events versus the axial source position.

\begin{tabular}{|c|c|c|c|c|c|c|}
\hline \multirow{3}{*}{$\begin{array}{l}\text { Point source axial } \\
\text { offset position }(\mathrm{cm})\end{array}$} & \multicolumn{6}{|c|}{ Radial and tangential resolution (mm FWHM) } \\
\hline & \multicolumn{2}{|c|}{$\mathrm{Si}-\mathrm{Si}$} & \multicolumn{2}{|c|}{$\mathrm{Si}-\mathrm{BGO}$} & \multicolumn{2}{|c|}{ BGO-BGO } \\
\hline & Radial & Tangential & Radial & Tangential & Radial & Tangential \\
\hline 0.0 & 0.37 & 0.34 & 1.01 & 1.01 & 1.73 & 1.66 \\
\hline 0.3 & 0.36 & 0.34 & 1.03 & 1.01 & 1.73 & 1.67 \\
\hline 0.6 & 0.36 & 0.34 & 1.01 & 1.00 & 1.72 & 1.67 \\
\hline 0.9 & 0.36 & 0.34 & 1.03 & 1.00 & 1.74 & 1.70 \\
\hline 1.2 & 0.36 & 0.33 & 1.00 & 1.01 & 1.73 & 1.71 \\
\hline 1.5 & 0.36 & 0.34 & 1.00 & 1.00 & 1.70 & 1.67 \\
\hline 1.8 & 0.37 & 0.34 & 1.01 & 1.01 & 1.72 & 1.68 \\
\hline
\end{tabular}

true coincidence is given by $T=g \varepsilon^{2} S$, where $S$ is the activity, $\varepsilon$ is the singles detection efficiency and $g$ is a geometric efficiency. Since the true coincidence rate is proportional to 


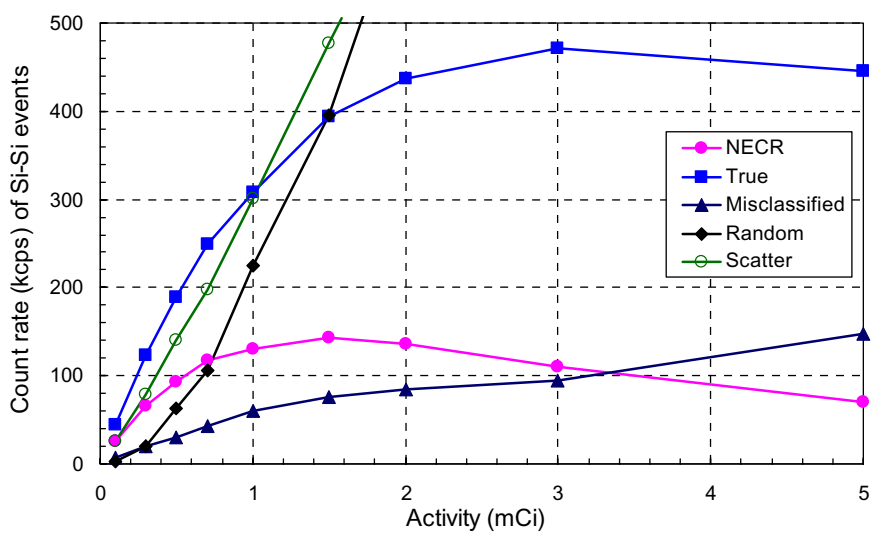

Figure 10. NECR, true, misclassified, random and scatter count rates of the Si-Si coincidence events as a function of source activity. An energy window (511-1533 keV) for total energy sum and $7 \mathrm{~ns}$ coincidence timing windows were used to measure coincidences.

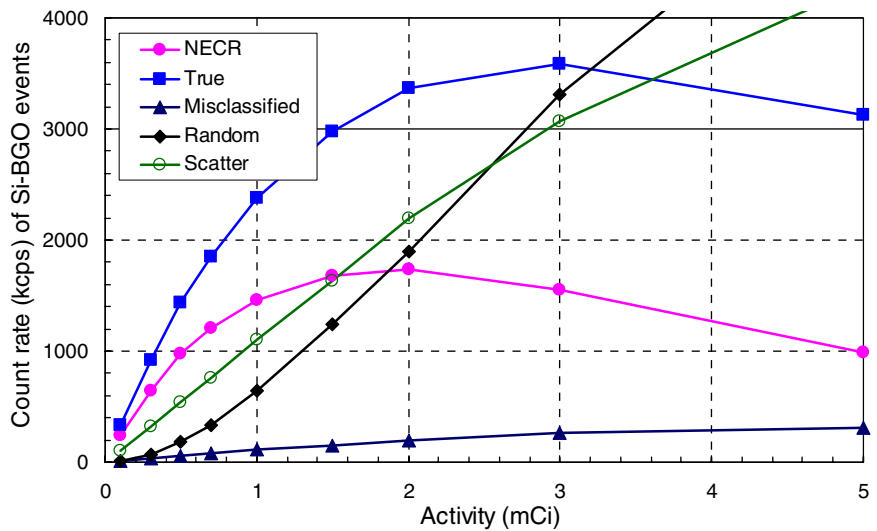

Figure 11. NECR, true, misclassified, random and scatter count rates of the Si-BGO coincidence events as a function of source activity. An energy window (511-1533 keV) for total energy sum and $7 \mathrm{~ns}$ coincidence timing windows were used to measure coincidences.

source activity, the rates of true coincidences of the $\mathrm{Si}-\mathrm{Si}, \mathrm{Si}-\mathrm{BGO}$ and BGO-BGO using a timing window of $7 \mathrm{~ns}$ increase until they reach the maximum rates at $3 \mathrm{mCi}$ of activity. Then the true rates decrease because the multiple events which are excluded from the true rates occurred in the timing window. These multiple events may be a potential problem in not only the proposed system but also in any other PET systems. However, they can be overcome with optimal operating conditions including the source activity and the width of the timing window estimated from a maximum NECR of the PET system.

The NECR, true, misclassified, random and scatter count rates of the $\mathrm{Si}-\mathrm{Si}, \mathrm{Si}-\mathrm{BGO}$ and BGO-BGO coincidence events as a function of source activity using $7 \mathrm{~ns}$ timing windows are plotted in figures 10-12. The peak NECR of the Si-Si events is $143 \mathrm{kcps}$ at $1.5 \mathrm{mCi}$ of activity. A maximum NECR of 1740 and $4333 \mathrm{kcps}$ is obtained with activity of $2 \mathrm{mCi}$ from $\mathrm{Si}-\mathrm{BGO}$ and BGO-BGO events, respectively. 


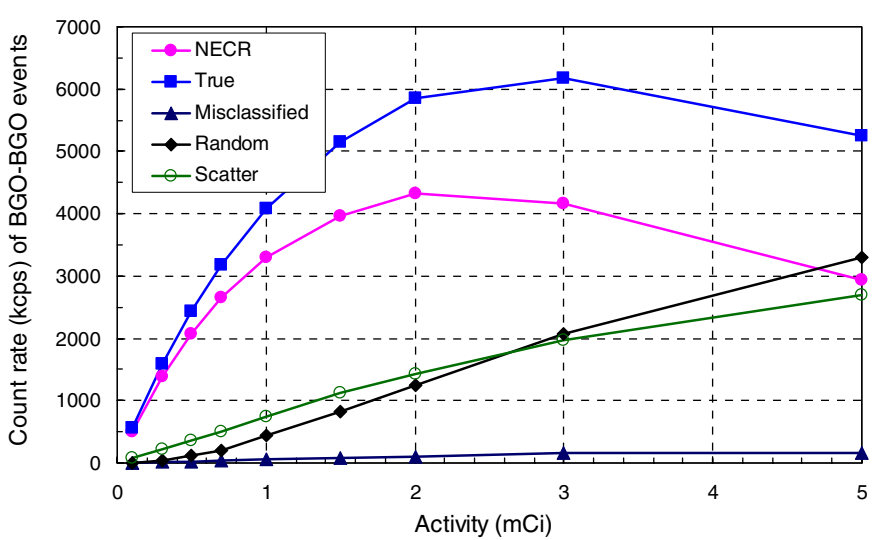

Figure 12. NECR, true, misclassified, random and scatter count rates of the BGO-BGO coincidence events as a function of source activity. An energy window $(511-1533 \mathrm{keV})$ for total energy sum and $7 \mathrm{~ns}$ coincidence timing windows were used to measure coincidences.

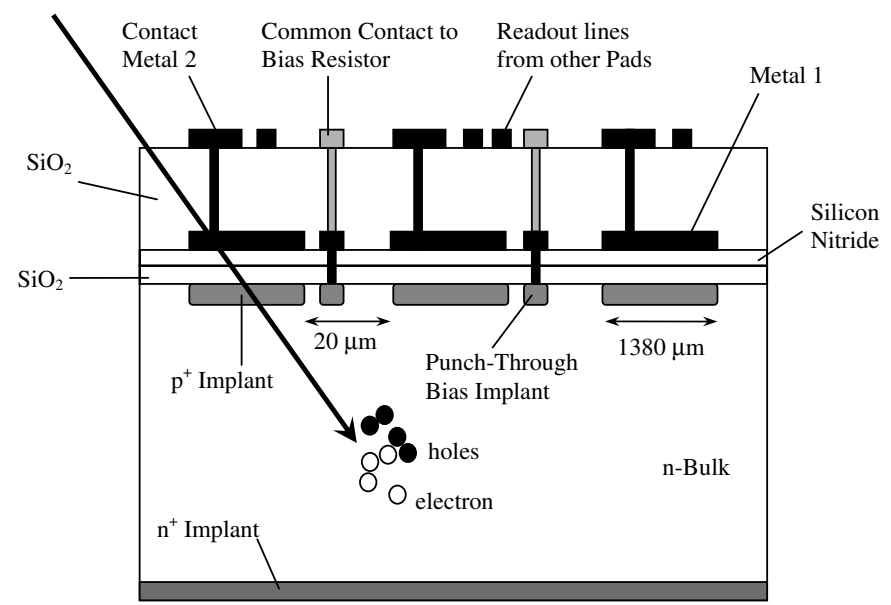

Figure 13. Cross-sectional view of a double-metal silicon pad sensor illustrates implants and metal layers (not to scale).

\section{Discussion}

There are a number of challenges presented by the design and construction of an actual system. We have constructed a prototype experimental system for a single-slice image instrument (see figures 13 and 14(a)) and demonstrated sub-millimeter spatial resolution $(\sim 700 \mu \mathrm{m}$ FWHM intrinsic detector resolution and $980 \mu \mathrm{m}$ reconstructed resolution) with very high-resolution events ( $\mathrm{Si}-\mathrm{Si}$ interactions) using two silicon pad detectors, each consisting of $5121.4 \mathrm{~mm}$ $\times 1.4 \mathrm{~mm} \times 1 \mathrm{~mm}$ pixels (Park et al 2007a). Recent experimental results were published in Phys. Med. Biol. (Park et al 2007b). In this section, we will discuss the challenges including production cost, detector packaging and cooling, timing, image reconstruction and event classification. 


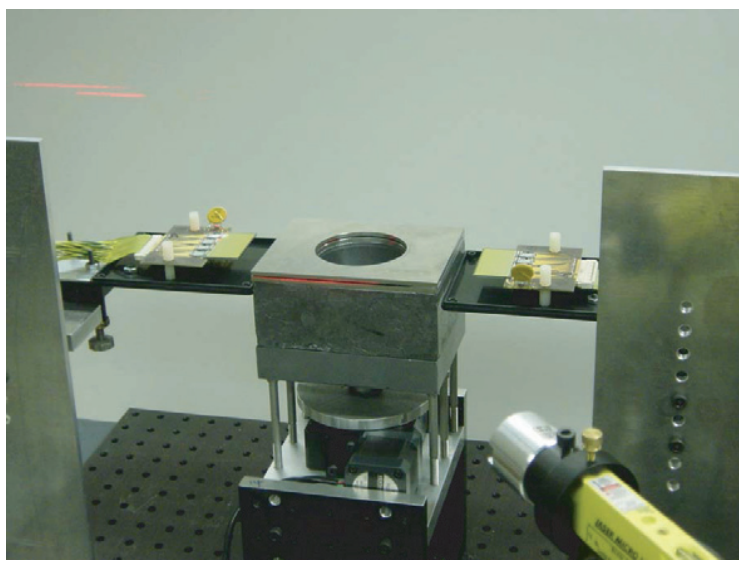

(a)

(c)

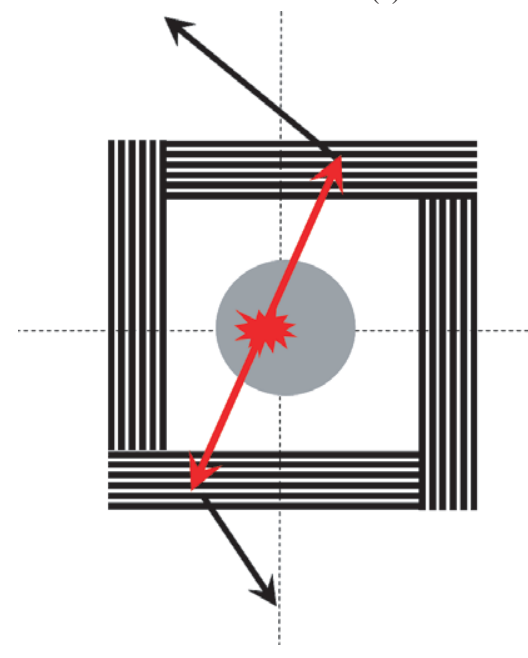

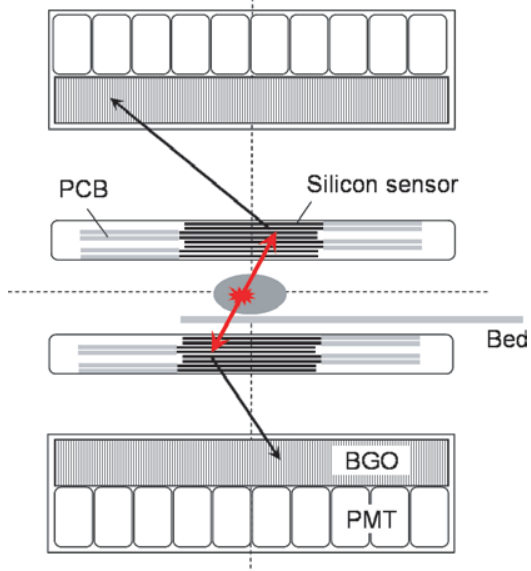

(b)

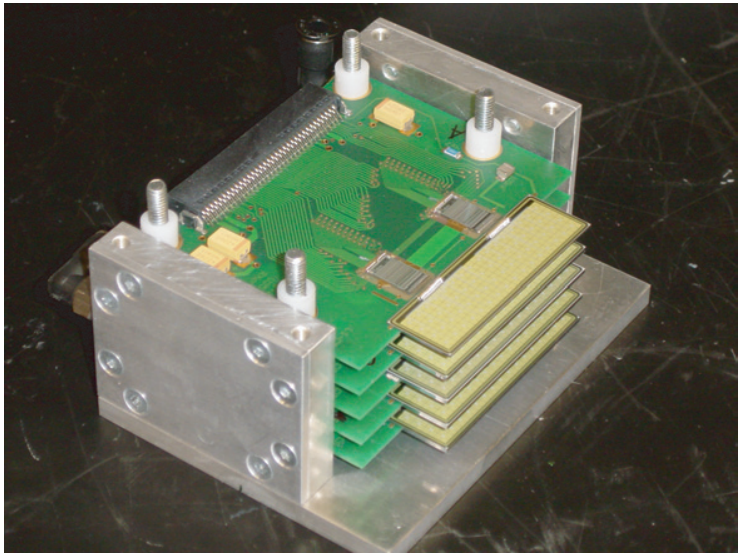

(d)

Figure 14. (a) A prototype experimental PET instrument consisting of two silicon pad detectors for single-slice imaging. (b) Cross-sectional view of a conventional BGO PET and silicon scatter detector insert (not to scale). (c) Transaxial view of the silicon detector (not to scale). The thickness of the silicon sensor and the number of detectors can be varied. Different shapes such as hexagon, octagon, and so on, can be used. Each detector will be surrounded by $\sim 1 \mathrm{~mm}$ guard-ring area on all sides. (d) A stack of silicon sensors and readout ASICs on PCBs.

\subsection{Production cost}

At the present low-volume prices, the cost of the inner detector ring and the associated readout electronics for the simulation of section $2.3(300 \mu \mathrm{m} \times 300 \mu \mathrm{m} \times 1 \mathrm{~mm}$ elements) would be approximately 1.8 million USD (1.5 USD/pad including readout $\times 1.2$ million pads). While cost will decrease dramatically with volume, there are a number of additional options for reducing cost involving reducing the number of individual detector channels requiring readout.

One of the more obvious ways to do this is to increase the size of the detector pads. Because of positron range, use of $300 \mu \mathrm{m} \times 300 \mu \mathrm{m}$ pads is likely overkill. Additional investigations 
are required to determine the pad size below which only marginal improvements in resolution can be achieved. Another option for reducing the number of channels is to use double-sided silicon strip detectors (DSSDs) rather than pad detectors (Weilhammer et al 1996, Knoll (1999) pp 493-7, Protic et al 2002). This can reduce $M \times N$ electronics channels to $M+N$. However, detector dead time and signal pileup at high count rates with DSSDs will introduce sensitivity and resolution loss. One might think also with DSSDs that ambiguities would be an issue at high event rates. However, even if there are multiple hits in a given interval, virtually all interactions are Compton scatters and will deposit different energies. The charge read out on both sides for each event must be the same within the noise. Therefore, it is possible to separate ambiguities in this manner. These effects are being studied to determine the suitability of DSSDs for this application. Finally, there are readout schemes for multiplexing position information in pad detectors. As an example, consider the pad detector as a checkerboard of red and black squares where only the red squares are connected to readout channels. The signal from each black square is capacitively coupled to its four red neighbors. The tradeoff in this approach, which is also under investigation, is increased noise.

\subsection{Detector packaging}

Figure 2 shows that the most probable angle of scatter in silicon for $511 \mathrm{keV}$ photons is about $35^{\circ}$. Since scatter fraction at angles less than $90^{\circ}$ is $70 \%$ of total scatter, the photon scattered in the silicon detector will have low probability to impinge and scatter in the mechanical support structure and readout electronics including printed circuit boards (PCB), tracers and application-specific integrated circuits (ASIC) located out of the axial FOV of the silicon ring (see figure 14(b)). However, it is true that construction materials, detector packaging materials, readout electronics, guard ring around the edge of the silicon detector can be a source of scatter. Although the low energy resolution of BGO restricts the total energy resolution, the energy sum and threshold still can be used to reject annihilation photons that have scattered through large angles in the object and detector mechanical support structure. Therefore, the detector sensitivity, spatial resolution and resolution uniformity will not be significantly affected by the scatters from the detector supporting materials. This was proved by experiment (Park et al 2007a, 2007b). With this reason, we did not include details such as construction materials, detector packaging materials and readout electronics in this design study.

Since conventional PET detector modules consist of large numbers of discrete small scintillation crystals, there are gaps between the crystals. The gaps were filled with a reflector material to optically isolate each crystal. Unlike a conventional PET module, the silicon detector has no gap between pixels. Figure 13 shows the cross-sectional view of the double-metal silicon pad sensor which was used for the experimental prototype. The gap $(20 \mu \mathrm{m})$ between the readout pads will not affect sensitivity.

Figures 14(b) and (c) illustrate a cross-sectional view of a system design consisting of a silicon scatter detector insert surrounded by a conventional BGO PET system and a transaxial view of a silicon detector, respectively. A stack of silicon sensors bonded to readout ASICs on PCBs is shown in figure 14(d). The gap between two silicon layers shown in figures 14(b), (c) and (d) will introduce some loss of packing fraction and coincidence sensitivity due to the loss of total effective silicon thickness. Our colleagues (Kagan et al 2006) have achieved 73\% packing fraction using a method of closely stacking the $1 \mathrm{~mm}$ thick 512-pad silicon sensors with $0.5 \mathrm{~mm}$ gaps (see figure 14(b)). By alternating the sides the PCBs are on, this can be extended as necessary with the same packing fraction. The gap between modules can probably be reduced below $0.5 \mathrm{~mm}$ using low-profile wirebonds or tape-automated bonding (TAB). Since we are trying to improve detector design and packing fraction by reducing the gap, we did 
not include the gap between silicon rings in this preliminary assessment of the proposed PET system. However, one can approximately estimate the loss of sensitivity using the plot of coincidence sensitivity as a function of silicon thickness in figure 3. Figures 13 and 14 give an idea how to mechanically extract readout channels out of the silicon ring. In addition, we have presented the signal readout sequence using the readout ASICs in Park et al (2007a).

Dense packaging of the silicon detectors and readout electronics present problems for both mechanical support and detector cooling. By using kapton hybrid tape with TAB techniques to connect the silicon sensor and readout electronics, silicon detectors can be densely packed and the heat from the electronics can be dissipated without heating the detectors. Electronic connections will be pulled out from detectors along the axis of the tomograph using kapton microcables which have $\sim 10 \mu \mathrm{m}$ thickness with about $20 \mu \mathrm{m}$ wide aluminum traces (Lacasta et al 2005). Therefore readout electronics can be placed further away from the sensor to reduce the amount of mass which causes scatter and attenuation for photons in the vicinity of the silicon sensor. The experimental study shows that the current silicon sensors closely wirebonded to readout chips (VATAGP3 ASIC from IDEAS) have excellent energy resolution $\left(\sim 1.4 \mathrm{keV}\right.$ FWHM at the $59.5 \mathrm{keV}$ photopeak of ${ }^{241} \mathrm{Am}$ and $140.5 \mathrm{keV}$ photopeak of $\left.{ }^{99 \mathrm{~m}} \mathrm{Tc}\right)$ due to the large number of information carriers (electron-hole pairs) coupled with low electronic noise. If one places readout ASICs away from sensors, the increased capacitance resulting from the increased trace length between sensors and ASICs will introduce electronic noise. Therefore, it is important to control the capacitance in order to reduce the impact of the length of the TAB microcables on the noise. It is not particularly clear how much additional noise will be introduced by moving the ASICs back with the microcables. However, the present detector noise we achieve is likely overkill. The triggering level on the present silicon detector can be set as low as $\sim 3 \mathrm{keV}$ with few noise pulses. It is not necessary to set this low threshold for PET. About 10-20 keV will result in acceptable losses in efficiency.

BGO detector gaps between modules will cause sensitivity loss but were not simulated in this study. However, the tapered pentagon photomultiplier-quadrant-sharing BGO detector technology proposed by Xie et al (2005) would provide nearly 100\% packing fraction for a ring type PET scanner.

\subsection{Timing resolution}

Good coincidence timing performance is required for the proposed PET scanner. This is particularly true for the four-fold coincidence required for this system. With low-noise charge sensitive amplifier chips (VA32C, a CMOS VLSI chip for 32 channel readout), fast triggering chips (TA32CG) have been developed for self-triggering signal readout of silicon pad detectors from integrated detector and electronics (Ideas ASA). The timing resolution from a TA32CG chip using a leading edge trigger is $17 \mathrm{~ns}$ FWHM due to significant time walk with the wide range of pulse height (Meier et al 2002). A new trigger chip being developed with time-walk compensation will significantly improve timing performance. Timing resolution for the scintillation detectors should be less than $10 \mathrm{~ns}$. Detector dead time and signal pileup at high count rates from high-activity sources may introduce additional loss of coincidence events. To evaluate these effects, accurate simulations of specific subsystems are presently being developed.

\subsection{Image reconstruction}

The PET system designed in this study generates three data sets with different resolutions and sensitivities. FBP separately reconstructs three different resolution images without weighting the signal-to-noise ratio from the datasets (see figure 9). However, these datasets can be 
combined into a single image using a maximum likelihood expectation maximization (MLEM) method to weight properly each dataset and retain high-frequency information (Shepp and Vardi 1982, Lange and Carson 1984, Kaufman 1993). Image reconstruction using a penalized ML-EM is described in Clinthorne et al (2003).

\subsection{Event classification}

Although the silicon detectors are capable of excellent energy resolution, the overall system energy resolution is limited by the second detector energy resolution, and no use is made of the silicon energy resolution capabilities in this system. However, this information can be used to improve system performance in three ways. (I) The Compton camera concept in the proposed PET system may be used to improve system performance. Since the Compton scattering angle can be estimated from the silicon energy and the position of the scattered photon is recorded by the second detector, one has independent information about the direction of the second annihilation photon such that it must lie within the backprojected conical shell. For this reason, the LORs of only true coincidences lie on the surface of the cone, but those of random and scatter events do not. Therefore, Compton kinematics information provides a method to significantly improve the NECR of the scanner by rejecting random coincidences and photon pairs in which either one or both have been scattered in the object (Park et al 2004). (II) In high-count-rate situations, the same information might be used to select a likely interaction point in the case of accidental coincidences or even to estimate the LOR by itself. (III) In the simple model, only events that scatter once in silicon and then escape are used for imaging since it is difficult to determine which interaction occurred first when there are multiple scatters. However, as shown by Kamae and Enomoto (1989) and Kroeger et al (2002), with good energy and position information it is possible to obtain good estimation of the interaction sequence for multiple scatter events. This could increase absolute sensitivity up to an additional $1.0 \%$ for $\mathrm{Si}-\mathrm{Si}$ and $3.0 \%$ for $\mathrm{Si}-\mathrm{BGO}$ events.

\section{Conclusions}

We have presented initial design and performance evaluations of a very high-resolution small animal PET scanner based on a solid-state silicon scatter detector having high energy and spatial resolution inserted into a conventional scintillator-based PET scanner. The design provides a potential solution for inter-crystal scatter and the DOI uncertainty problem in conventional animal PET scanners using scintillation materials. Sub-millimeter spatial resolution of $340 \mu \mathrm{m}$ FWHM at the center of the FOV with $1 \%$ sensitivity is predicted from the coincidence of single Compton scattering events in the $300 \mu \mathrm{m} \times 300 \mu \mathrm{m} \times 1$ $\mathrm{mm}$ silicon pad detectors. The high resolution of $\sim 1 \mathrm{~mm}$ FWHM with $9 \%$ sensitivity was additionally predicted from the coincidence of single scattering in the silicon and full energy absorption of the second photon in the BGO detector.

In spite of the potentially high performance of the proposed instrument, a number of significant challenges-most relating to construction of the inner detector ring-remain. In order to investigate these challenges, we are currently conducting additional simulations and developing experimental systems.

\section{Acknowledgments}

This work was supported by the United States National Institutes of Health under grants R01 EB000430-31 and R41 RR15133-01. 


\section{References}

Bielajew A F and Rogers D W O 1987 PRESTA: the parameter reduced electron-step transport algorithm for electron Monte Carlo transport Nucl. Instrum. Methods Phys. Res. B $18165-81$

Burr K C et al 2004 Evaluation of a prototype small-animal PET detector with depth-of-interaction encoding IEEE Trans. Nucl. Sci. 51 1791-8

Clinthorne N H et al 2000 Very high resolution animal PET J. Nucl. Med. 4120

Clinthorne N H et al 2003 Multi-resolution image reconstruction for a high-resolution small animal PET device IEEE Nucl. Sci. Symp. Med. Imaging Conf. 3 1197-2001

Comanor et al 1996 Algorithms to identify detector Compton scatter in PET modules IEEE Trans. Nucl. Sci. $432213-8$

Derenzo S E 1979 Precision measurement of annihilation point spread distributions for medically important position emitters 5th Int. Conf. Positron Annihilation (Sendai, Japan)

Derenzo S E 1986 Mathematic removal of positron range blurring in high resolution tomography IEEE Trans. Nucl. Sci. 33 565-9

Dokhale P A et al 2006 Intrinsic spatial resolution and parallax correction using depth-encoding PET detector modules based on position-sensitive APD readout IEEE Trans. Nucl. Sci. 53 2666-70

Evans R D 1995 The Atomic Nucleus (New York: McGraw Hill) pp 629-30

Haber S F et al 1990 Application of mathematical removal of positron range blurring in positron emission tomography IEEE Trans. Nucl. Sci. 37 1293-9

Harrison R L et al 1999 Positron range and coincidence non-collinearity in SimSET IEEE Nucl. Sci. Symp. Med. Imaging Conf. 3 24-30

Huber J S et al 2001 An LSO scintillator array for a PET detector module with depth of interaction measurement IEEE Trans. Nucl. Sci. 48 684-8

Janecek M et al 2006 A simulation study for the design of a prototype insert for whole-body PET scanners IEEE Trans. Nucl. Sci. $\mathbf{5 3} 1143-9$

Kagan H et al 2006 New developments in Compton SPECT imaging EuroMedIm Conf. Proc. (Marseille, France, 9-12 May 2006)

Kamae T and Enomoto R 1989 Gamma ray measurement utilizing multiple Compton scattering US Patent 4857737

Kaufman L 1993 Maximum likelihood reconstruction for emission tomography IEEE Trans. Med. Imaging 12 200-14

Knoll G F 1999 Radiation Detection and Measurement 3rd edn (New York: Wiley)

Kroeger R A et al 2002 Three-Compton telescope: theory, simulations, and performance IEEE Trans. Nucl. Sci. 49 1887-92

Lacasta C et al 2005 Development and test of TAB bonded micro-cables for silicon detectors in a Compton prostate probe IEEE Nucl. Sci. Symp. Med. Imaging Conf. 5 23-9

Lange K and Carson R 1984 EM reconstruction algorithms for emission and transmission tomography J. Comp. Assist. Tomogr. 8 306-16

Levin C S and Hoffman E J 1999 Calculation of positron range and its effect on the fundamental limit of positron emission tomography system spatial resolution Phys. Med. Biol. 44 781-99

Meier D et al 2002 Silicon detector for a Compton camera in nuclear medical imaging IEEE Trans. Nucl. Sci. 49 812-6

Michaud J B et al 2004 Experimental results of identification and vector quantization algorithms for DOI measurement in digital PET scanner with phoswich detectors IEEE Nucl. Sci. Symp. Med. Imaging Conf. 6 3678-81

Miyaoka R S and Lewellen T K 2000 Effect of detector scatter on the decoding accuracy of DOI detector module IEEE Trans. Nucl. Sci. 47 1614-9

Miyaoka R S et al 1998 Design of depth of interaction (DOI) PET detector module IEEE Trans. Nucl. Sci. 45 1069-73

Nelson W R et al 1985 The EGS4 code system SLAC-Report-265

Palmer M R and Brownell G L 1992 Annihilation density distribution calculations for medically important position emitters IEEE Trans. Med. Imaging $11373-8$

Park S et al 2001a Design of a very high resolution animal PET J. Nucl. Med. 4255

Park S et al 2001b Experimental setup for very high resolution animal PET based on solid state detector IEEE Nucl. Sci. Symp. Med. Imaging. Conf. 2 1120-3

Park S et al 2002 Effect of recoil electron range on sensitivity and on spatial resolution of very high resolution animal PET IEEE Nucl. Sci. Symp. Med. Imaging Conf. 3 1756-9

Park S et al 2003 Effect of inter-crystal Compton scatter on efficiency and image noise in small animal PET module IEEE Nucl. Sci. Symp. Med. Imaging. Conf. 4 2272-7

Park S et al 2004 Improvement of noise equivalent count rate using Compton kinematics in a Compton PET IEEE Nucl. Sci. Symp. Med. Imaging Conf. 6 3911-5

Park S et al 2007a A prototype of very high-resolution small animal PET scanner using silicon pad detectors Nucl. Instrum. Methods Phys. Res. A 570 543-55 
Park S et al 2007b Performance evaluation of a very high resolution small animal PET images using silicon scatter detectors Phys. Med. Biol. 52 2807-26

Protic D et al 2002 Development of double-sided microstructured Si(Li) detectors IEEE Trans. Nucl. Sci. 49 1993-8

Qi J et al 1998 High-resolution 3D Bayesian image reconstruction using the microPET small-animal scanner Phys. Med. Biol. 43 1001-13

Seidel J et al 2003 Resolution uniformity and sensitivity of NIH ATLAS small animal PET scanner: comparison to simulated LSO scanners without depth-of-interaction capability IEEE Trans. Nucl. Sci. 50 1347-50

Shao Y et al 1996 A study of inter-crystal scatter in small scintillator arrays designed for high resolution PET imaging IEEE Trans. Nucl. Sci $\mathbf{4 3}$ 1938-44

Shao Y et al 2002 Dual APD array readout of LSO crystals: optimization of crystal surface treatment IEEE Trans. Nucl. Sci. 49 649-54

Shepp L A and Vardi Y 1982 Maximum likelihood reconstruction for emission tomography IEEE Trans. Med. Imaging $1113-22$

Tai Y C et al 2003 Design study of a detector insert for high resolution clinical PET imaging IEEE Nucl. Sci. Symp. Med. Imaging Conf. 3 1714-7

Weilhammer P et al 1996 Si pad detectors Nucl. Instrum. Methods Phys. Res. 383 89-97

Wilderman S J 1990 Vectorized algorithms for Monte Carlo simulations of kilovolt electron and photon transport PhD Dissertation, Department Of Nuclear Engineering, University of Michigan, Ann Arbor, MI

Xie S et al 2005 A pentagon photmultiplier-quadrant-sharing BGO detector for a rodent research PET (RRPET) IEEE Trans. Nucl. Sci. 52 210-6 